\title{
Analysis of RIS-Based Terrestrial-FSO Link over G-G Turbulence with Distance and Jitter Ratios
}

\author{
Alain R. Ndjiongue, Senior Member, IEEE, Telex M. N. Ngatched, Senior Member, IEEE, Octavia A. Dobre, \\ Fellow, IEEE, Ana G. Armada, Senior Member, IEEE, and Harald Haas, Fellow, IEEE
}

\begin{abstract}
One of the main problems faced by communication systems is the presence of skip-zones in the targeted areas. With the deployment of the fifth-generation mobile network, solutions are proposed to solve the signal loss due to obstruction by buildings, mountains, and atmospheric or weather conditions. Among these solutions, reconfigurable intelligent surfaces (RIS), which are newly proposed modules, may be exploited to reflect the incident signal in the direction of dead zones, increase communication coverage, and make the channel smarter and controllable. This paper tackles the skip-zone problem in terrestrial free-space optical (T-FSO) systems using a single-element RIS. Considering link distances and jitter ratios at the RIS position, we carry out a performance analysis of RIS-aided TFSO links affected by turbulence and pointing errors, for both heterodyne detection and intensity modulation-direct detection techniques. Turbulence is modeled using the Gamma-Gamma distribution. We analyze the model and provide exact closedform expressions of the probability density function, cumulative distribution function, and moment generating function of the end-to-end signal-to-noise ratio. Capitalizing on these statistics, we evaluate the system performance through the outage probability, ergodic channel capacity, and average bit error rate for selected binary modulation schemes. Numerical results, validated through simulations, obtained for different RIS positions and link distances ratio values, reveal that RIS-based T-FSO performs better when the RIS module is located near the transmitter.
\end{abstract}

Index Terms-Free-space optical communications, reconfigurable intelligent surfaces, unified Gamma-Gamma turbulence channels with pointing errors, average bit error rate, ergodic channel capacity, outage probability.

\section{INTRODUCTION}

The recent extensive investigation of optical wireless communications in the outdoor environment, also called free-space optical (FSO), is motivated by its advantages compared to its radio frequency $(\mathrm{RF})$ counterpart, especially in point-to-point networks. These advantages include larger bandwidth, higher channel capacity, and cost-effectiveness due to an unlicensed environment [1], which can be leveraged to solve the bandwidth limitation in the RF technology. Its most prominent applications are satellite-to-ground, satellite-to-satellite, and terrestrial FSO (T-FSO) systems such as building-to-building $(\mathrm{B} 2 \mathrm{~B})^{1}$ communications. Besides turbulence, pointing errors,

Alain. R. Ndjiongue, T. M. N. Ngatched, and O. A. Dobre are with the Faculty of Engineering and Applied Science, Memorial University of Newfoundland, Canada.

Ana G. Armada is with the Signal Theory and Communications Department, Universidad Carlos III de Madrid, Spain.

Harald Haas is with the LiFi Research and Development Center, Department of Electronic and Electrical Engineering, the University of Strathclyde, Glasgow, United Kingdom.

${ }^{1} \mathrm{~A}$ B2B environment is a T-FSO data transmission environment where the information is transferred between buildings. and attenuation that affect optical signals over the FSO channel, signal obstruction due to buildings or trees can prevent the transmitted wave from reaching the intended destination. We attempt to solve this obstruction's problem in T-FSO systems, affected by moderate-to-strong turbulence levels and pointing errors, using reconfigurable intelligent surfaces (RIS). RIS are electromagnetic devices with electronically controllable characteristics. They can reflect, refract, extinct, or scatter the incoming signal to impact its amplitude, phase, and polarization. The design of RIS modules depends on the application.

The RIS module is a planar array of multiple mirrors or optical phased-array structures, used to guide the incoming signal toward a targeted area and reconfigure the transmission channel [2]. It offers wireless networks several advantages over competing technologies such as relay systems. In addition to its low power consumption, the RIS module is made of electronically controllable elements. These advantages have recently triggered intensive and extensive investigations of the technology. It has lately been proposed to solve the dead zone problems in RF networks and create smart communication channels and environments $[3]-[5]$. As part of the channel, the RIS elements may decisively impact wireless communication systems' performance, leading to the need for new pre-coding designs [6]. The RIS concept has been extended to optical systems [7]-[14], reconfigurable optical components [15] and hybrid systems [16], [17]. In [7] and [8], the RIS module is placed inside the visible light communications (VLC)'s receiver and serves as a wave-guard to steer and/or amplify the incoming light, resulting in an improved receiver fieldof-view and transmission range. On the contrary, in [9], the authors evaluated the impact of a diffuse, specular, and glossy roof-type RIS on an indoor VLC link, while in [10], two types of indoor VLC RIS structures were proposed and analyzed.

The investigation of using RIS in T-FSO systems is still in its infancy; however, it is attracting significant research interest. Up to date, only a few ideas have been proposed for the free-space environment using an optical signal [11][14], [18]. Early work on employing a RIS module in FSO systems is proposed in [11], where the authors introduced transmission in RIS-based FSO systems. The system and channel models are evaluated and the RIS-based FSO system's geometry is presented. The authors also analyzed the pointing displacement, leading to a system statistical model with a focus on 2-D and 3-D representations. This work proposed conditional geometric and misalignment losses (GML) versus misalignment for different RIS sizes. Finally, the work derived the probability density function (PDF) of the GML versus the 


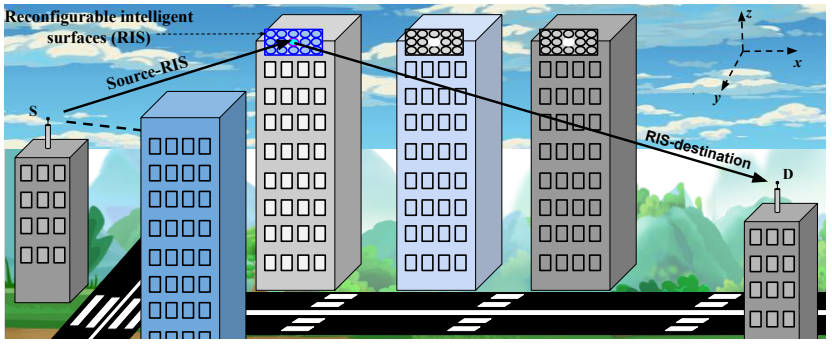

Fig. 1: The proposed system.

channel gain for the 2-D and 3-D scenarios. In [12], which is an extension of [11], the authors showed more steps and insight in deriving the PDF of the GML for the RIS-based FSO link. They illustrated the RIS phase-shift matrix and proposed a general expression of the PDF of the pointing misalignment. This PDF is combined with two turbulence models, namely log-normal and Gamma-Gamma (G-G), to carry out the outage probability (OP) analysis of the proposed system. The pointing error channel response results are given versus normalized misalignment and multiple RIS sizes. In [13] the authors exploited an unmanned aerial vehicle to design a RIS-based FSO system, while in [14], the authors developed an analytical end-to-end channel model for RISassisted FSO systems based on the Huygens-Fresnel principle. Results of the pointing displacement in terms of the lens $x-y$ coordinates are presented. Lastly, the paper provided the bit error rate (BER) versus the RIS-receiver distance and geometric far-field approximation. In [18], a power-amplifying RIS was proposed to solve the double fading affecting the FSO communication system. In [19], the authors discussed the implementation of a RIS-based FSO system considering controllable multi-branches, while in [20], based on the central limit theorem, the authors exploited the Gaussian distribution to approximate a G-G channel for many transmitting signals.

Due to the presence and locations of obstacles, the use of RIS in FSO is suitable for T-FSO communication systems such as $\mathrm{B} 2 \mathrm{~B}$, which represents the environment of interest for our work. We consider a T-FSO environment in which the source (S) and the destination (D) can not be linked through a lineof-sight. We consider a single-element RIS with the phaseshift profile model proposed in [12], and with an adjustable reflective plane over its central axis. Contrarily to the works in [11] and [12], we analyze the RIS-based T-FSO system using a unified and combined expression of the T-FSO link's PDF based on the Meijer-G function and obtain closed-form expressions for numerous metrics and several positions of the RIS.

To the best of our knowledge, analyzing a RIS-based T-FSO system characterized by G-G turbulence with pointing errors, exploiting the unified expression of the end-to-end SNR's PDF, and providing results based on the S-RIS/RIS-D, pointing error ratios, and RIS positions, has not yet been proposed in the open literature and represents the motivation of this paper. The main goal of the paper is to show the numerical analysis and performance of the considered T-FSO system. To this end, we make the following contributions:

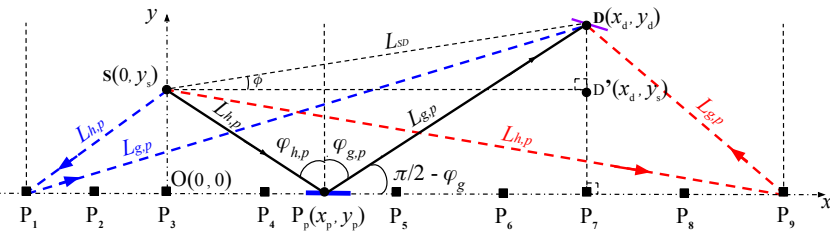

Fig. 2: Proposed system's model. $P_{x}$ denotes the $x^{\text {th }}$ position of the RIS module, $L_{S D}$ is the distance from source (S) to destination (D), $L_{h, p}$ and $L_{g, p}$ are respectively the distances from $\mathrm{S}$ to RIS and RIS to D, $\varphi_{h, p}$ and $\varphi_{g, p}$ respectively represent the angles of incidence and reflection of the transmitted beam central ray.

(i) First, at each position, $p$, of the RIS module, we study signal reflection at the RIS, determine the beam waists, $W_{z,(h, p)}$ and $W_{z,(g, p)}$, at the RIS and D, find pointing displacement standard deviations, $\xi_{h, p}$ and $\xi_{g, p}$, at the RIS and $\mathrm{D}$, respectively, and calculate their ratio, $k_{p}=\xi_{g, p} / \xi_{h, p}$. These are used to plot $W_{z,(h, p)}=f\left(\omega_{p}\right)$ and $W_{z,(g, p)}=f\left(\omega_{p}\right)$. Based on this analysis, we also plot $k_{p}=f\left(\omega_{p}\right)$, with $\omega_{p}$ as the distance ratio S-RIS/RIS-D.

(ii) Second, we derive closed-form unified statistical expressions of the PDF, cumulative distribution function (CDF), and moment generating function (MGF) of the end-to-end SNR.

(iii) Third, based on these closed-form statistics, we derive the OP, $P_{\text {out }}$, the average ergodic channel capacity, $\bar{C}$, and the average BER, $\bar{P}_{b}$, for selected binary modulation schemes, including coherent binary frequency-shift keying (CBFSK), non-coherent binary frequency-shift keying (NBFSK), coherent binary phase-shift keying (CBPSK), and differential binary phase-shift keying (DBPSK), and present results versus SNR and $\omega_{p}$, at several positions of the RIS module.

(iv) In addition, we derive closed-form expressions of the diversity order and coding gain for the proposed RIS-based T-FSO.

(v) Finally, we present numerical results, validated through simulations and asymptotic curves, for different SNR values and $\omega_{p}$, at several positions of the RIS module. These results enable us to get more insights on the S-RIS and RIS-D distance, and lead to the conclusion that a RIS-based T-FSO performs better when S-RIS < RIS-D.

\section{System AND Channel Models}

\section{A. System Model}

The environment under study is a cascaded system of a single light beam traveling from $\mathrm{S}$ to $\mathrm{D}$ after a reflection on a RIS element, as shown in Fig. 11 There is no direct link between S and D due to obstructions. The RIS module, located at the top of a building, serves as a reflector to the incoming light and ensures that the transmitted light points to the receiver. To achieve this, the RIS controller can act on the reflected angle through its phase-shift profile while the reflective plane is fixed or vice-versa. In this paper, we control the phase-shift profile and adjust the RIS lateral orientation at each position of the RIS, as shown in Fig. 2. We assume that both channel sections, which will be referred to as system subchannels, exhibit moderate-to-strong turbulence levels, and 


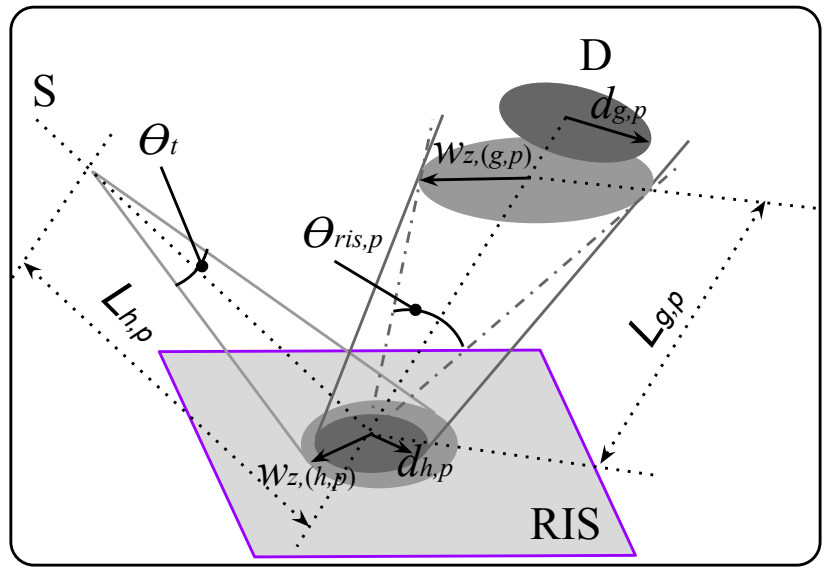

Fig. 3: Virtual beam divergence at RIS.

that the received signal is affected by pointing errors at the RIS and D, influenced by sways at S, the RIS, and D. The system is characterized by the RIS position dependent S-RIS and RISD distances, $L_{h, p}$ and $L_{g, p}$, respectively, the beam aperture diameters at the RIS and D, $d_{h, p}$ and $d_{g, p}$, respectively, the beam divergences at the RIS and D, $\theta_{t}$ and $\theta_{\text {ris, } p}$, respectively, as shown in Fig. 3. the beam waists at the RIS and D, $W_{z,(h, p)}$ and $W_{z,(g, p)}$, respectively, and the incidence angle (central ray) of the beam at the RIS, $\varphi_{h, p}$. At the receiver, the PD is oriented in such a way to form an angle, $\varphi_{g}$, with the incident light (central ray). The two sub-channels, $h$ and $g$, are characterized by their corresponding G-G turbulence atmospheric $\alpha-\beta$ parameters, $\alpha_{h, p}-\beta_{h, p}$ and $\alpha_{g, p}-\beta_{g, p}$, respectively, for the $h$ and $g$ sub-channels and varying with the RIS position. Scintillation in the system, reinforced by sways at S, the RIS, and D, creates pointing errors at the RIS and D, leading to increased effects of misalignment at the RIS and D [11], [12]. This source of errors is generally represented by the ratio of equivalent beam radius and pointing error displacement standard deviation, which at the RIS and D, are evaluated by $\xi_{h, p}$ and $\xi_{g, p}$, respectively, coupled to their corresponding jitter variances, $\rho_{h, p}$ and $\rho_{g, p}$, respectively. To characterize the transmission system, we define the ratios $k_{p}=\xi_{g, p} / \xi_{h, p}$ and $\omega_{p}=L_{h, p} / L_{g, p}$.

\section{B. Channel Model}

Generally, the FSO link is subject to three main signal impairment factors: attenuation, pointing errors, and atmospheric turbulence. These impairment sources, each in its way, affect the transmitted optical signal, $I$, and can be expressed as $I=I_{l} I_{m} I_{a}$, where $I_{l}, I_{m}$, and $I_{a}$ represent the received intensity affected by attenuation, pointing errors, and atmospheric turbulence, respectively.

1) Attenuation: The path loss, which is considered constant for a given weather condition and link distance [1], is given by the Beer-Lambert's law as $I_{l, i}=e^{-\delta_{i} L_{i}}$, where $\delta_{i}$ is the attenuation factor in the $i^{\text {th }}$ link. The overall attenuation can be expressed as $I_{l, p}=\Delta e^{-L_{h, p}\left(\delta_{h}+\delta_{g} / \omega_{p}\right)}$. The quantity $\Delta$ includes the aperture area, the divergence angle, the link distance, and other transmitter and receiver parameters such

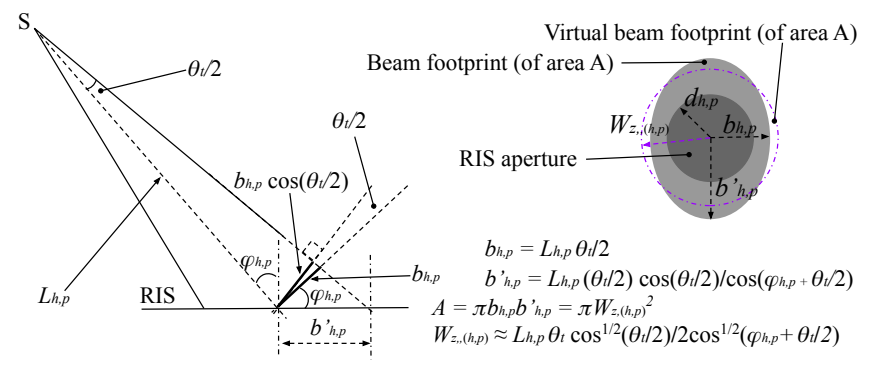

Fig. 4: Virtual beam footprint at the RIS.

as the concentration and conversion coefficients. For systems where $\delta_{h}=\delta_{g}, I_{l, p}$ reduces to

$$
I_{l, p}=\Delta e^{-L_{h, p} \delta_{h}\left(\frac{1+\omega_{p}}{\omega_{p}}\right)} .
$$

2) Pointing Errors: Figure 2 describes the path of the incoming beam through the RIS module. It is assumed that only the RIS aperture reflects the light and that the beam footprint on the RIS surface is bigger than the RIS aperture surface. The PDF describing pointing errors at the RIS and D, $f_{m,(i, p)}\left(I_{m,(i, p)}\right)$, is given by [1, Eq. (3)], [21]

$$
f_{m,(i, p)}\left(I_{m,(i, p)}\right)=\frac{\xi_{i, p}^{2}}{A_{o}^{\xi_{i, p}^{2}}} I_{m,(i, p)}^{\xi_{i, p}^{2}-1}, 0 \leq I_{m,(i, p)} \leq A_{0,(i, p)}
$$

where $i \in\{h, g\}, \xi_{i}$ is the ratio of equivalent beam radius to pointing displacement standard deviation at the RIS and D, $A_{o,(i, p)}=\left[\operatorname{erf}\left(v_{(i, p)}\right)\right]^{2}[1]$, with $\operatorname{erf}(\cdot)$ as the error function at the RIS and D, and $v_{(i, p)}=d_{i} \sqrt{\pi} / \sqrt{2} W_{z,(i, p)}$ [22], [23]. Here, $d_{i}$ and $W_{z,(i, p)}$ are the radius of the receiver aperture and the beam waist, respectively [1], [21]. By definition, the ratio between the equivalent beam radius to the pointing displacement standard deviation at the RIS and D is given by [22]-24]

$$
\xi(i, p)=\frac{W_{\mathrm{eq},(i, p)}}{2 \rho_{(i, p)}},
$$

where $\rho_{(i, p)}$ is the jitter variance at the RIS and D, and $W_{\text {eq, }(i, p)}$ is defined by [22], [23]

$$
W_{\mathrm{eq},(i, p)}=\frac{W_{z,(i, p)}^{2} \sqrt{\pi} \operatorname{erf}\left(v_{(i, p)}\right)}{2 v_{i} e^{-v_{(i, p)}^{2}}},
$$

where $W_{z,(i, p)}$ is the beam waist at the RIS/D and $d_{i}$ is the RIS and receiver aperture.

\section{- Beam waist at the RIS}

As shown in Fig. 4, the beam footprint on the RIS surface is not circular, but ellipsoidal with two main radii, $b_{h, p}$ and $b_{h, p}^{\prime}$, which can be approximated as $b_{h, p} \approx L_{h, p} \theta_{t} / 2$ and $b_{h, p}^{\prime}=L_{h, p} \theta_{t} \cos \left(\theta_{t} / 2\right) / 2 \cos \left(\varphi_{h, p}+\theta_{t} / 2\right)$. To evaluate the misalignment at the RIS, we consider about an equivalent circular virtual footprint with the same area as the ellipsoidal disk. This leads to a virtual beam waist expressed as $W_{z,(h, p)}=$ $L_{h, p} \theta_{t} \sqrt{\cos \left(\theta_{t} / 2\right)} / 2 \sqrt{\cos \left(\varphi_{h, p}+\theta_{t} / 2\right)}$. By substituting this 


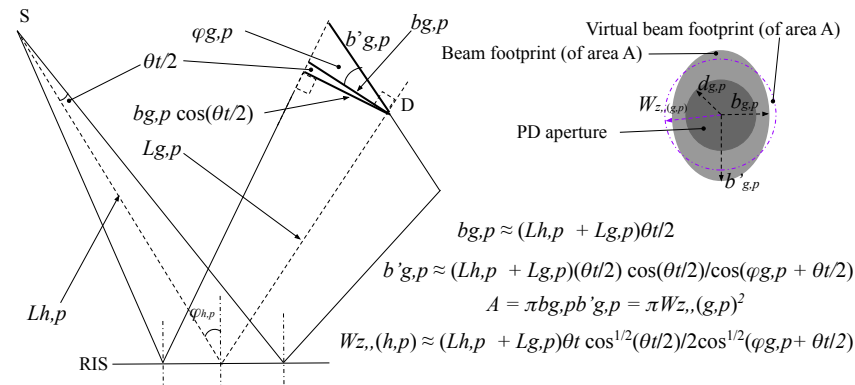

Fig. 5: Virtual beam footprint at D.

expression of $W_{z,(h, p)}$ into the expression of $v_{i, p}$ and using (4), we obtain the equivalent beam waist at the RIS given by

$$
W_{\mathrm{eq},(h, p)}=\frac{L_{h, p}^{3} \theta_{t}^{3} \cos ^{3 / 2}\left(\frac{\theta_{t}}{2}\right) \operatorname{erf}\left[\frac{2 d_{h} \sqrt{\pi \cos \left(\varphi_{h, p}+\frac{\theta_{t}}{2}\right)}}{L_{h, p} \theta_{t} \sqrt{2 \cos \left(\frac{\theta_{t}}{2}\right)}}\right]}{8 \sqrt{2} d_{h} \cos ^{3 / 2}\left(\varphi_{h}+\frac{\theta_{t}}{2}\right) \exp \left[-\frac{2 d_{h}^{2} \pi \cos \left(\varphi_{h, p}+\frac{\theta_{t}}{2}\right)}{L_{h, p}^{2} \theta_{t}^{2} \cos \left(\frac{\theta_{t}}{2}\right)}\right]} .
$$

\section{- Beam waist at $\mathrm{D}$}

As shown in Fig. 5. the beam footprint at the destination is also not circular, but an ellipsoidal disk with two main radii, $b_{g, p}$ and $b_{g, p}^{\prime}$. The receiver perceives the light as if it was generated at RIS with a divergence $\theta_{r i s, p}=\theta_{t}\left(1+\omega_{p}\right)$ (see Fig. 3). Due to the assumption on small-angle sine, they can be expressed as $b_{g, p} \approx\left(L_{g, p}+L_{h, p}\right) \theta_{t} / 2$ and $b_{g, p}^{\prime} \approx\left(L_{g, p}+L_{h, p}\right)\left(\theta_{t} / 2\right) \cos \left(\theta_{t} / 2\right) / \cos \left(\varphi_{g, p}+\theta_{t} / 2\right)$. To evaluate the misalignment at $\mathrm{D}$, we consider an equivalent virtual footprint with the same area as the ellipsoidal disk. This leads to a virtual beam waist expressed as $W_{z,(g, p)}=\left(L_{g, p}+\right.$ $\left.L_{h, p}\right) \theta_{t} \sqrt{\cos \left(\theta_{t} / 2\right)} / 2 \sqrt{\cos \left(\varphi_{g, p}+\theta_{t} / 2\right)}$. By substituting this expression of $W_{z,(g, p)}$ into the expression of $v_{i}$ and utilizing (4), we obtain the equivalent beam waist at $\mathrm{D}$, and replacing $\theta_{r i s, p}$ and $L_{g, p}$ by their expressions using $L_{h, p}$ and $\omega_{p}$ leads to

$$
\begin{aligned}
& W_{\mathrm{eq},(g, p)}= \\
& \frac{L_{h, p}^{3} \theta_{t}^{3}\left[\frac{\omega_{p}+1}{\omega_{p}}\right]^{3} \cos ^{3 / 2}\left(\frac{\theta_{t}}{2}\right) \operatorname{erf}\left[\frac{2 d_{g} \sqrt{\pi \cos \left(\varphi_{g, p}+\frac{\theta_{t}}{2}\right)}}{L_{h, p} \theta_{t}\left[\frac{\omega_{p}+1}{\omega_{p}}\right] \sqrt{2 \cos \left(\frac{\theta_{t}}{2}\right)}}\right]}{8 \sqrt{2} d_{g} \cos ^{3 / 2}\left(\varphi_{g, p}+\frac{\theta_{t}}{2}\right) \exp \left[-\frac{2 d_{g}^{2} \pi \cos \left(\varphi_{g, p}+\frac{\theta_{t}}{2}\right)}{L_{h, p}^{2} \theta_{t}^{2}\left[\frac{\omega_{p+1}}{\omega_{p}}\right]^{2} \cos \left(\frac{\theta_{t}}{2}\right)}\right]} .
\end{aligned}
$$

Let $k_{p}$ be the ratio of $\xi_{g, p}$ to $\xi_{h, p}$. It is expressed as

$$
k_{p}=\frac{\rho_{h, p}}{\rho_{g, p}} \frac{d_{h}}{d_{g}}\left(\frac{1+\omega_{p}}{\omega_{p}}\right)^{3}\left[\frac{\cos \left(\varphi_{h, p}+\frac{\theta_{t}}{2}\right)}{\cos \left(\varphi_{g, p}+\frac{\theta_{t}}{2}\right)}\right]^{3 / 2} \eta \mu,
$$

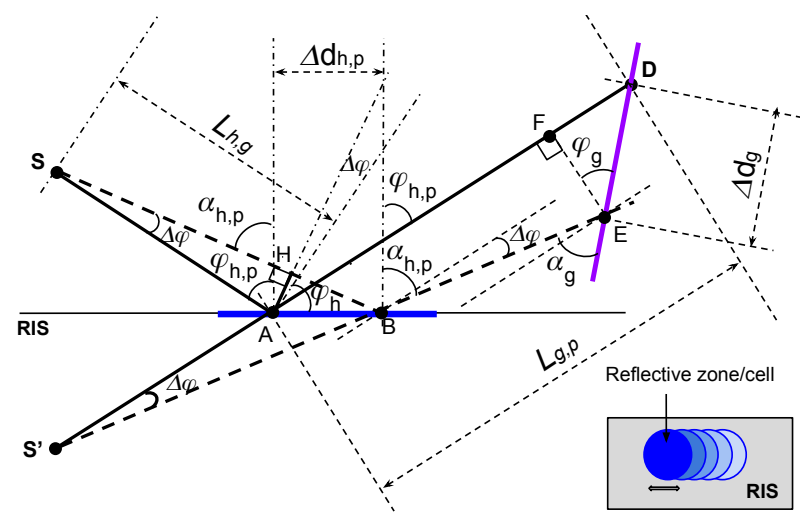

Fig. 6: Geometrical analysis of pointing misalignment at the RIS and D. SA, SB, AD, and BE are the rays at the center of the corresponding beam. The bottom-right part of the figure shows how the RIS's position can be shifted to optimize transmission.

where $\eta$ and $\mu$ are respectively given by

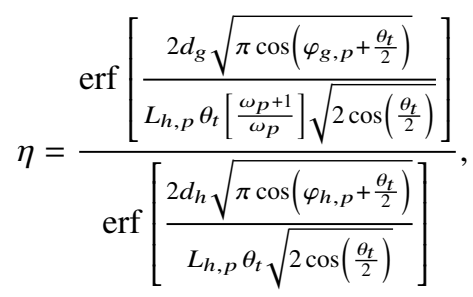

and

$$
\mu=e^{\left[\frac{2 \pi}{L_{h}^{2} \theta_{t}^{2} \cos \left(\frac{\theta_{t}}{2}\right)}\left(\frac{d_{g}^{2} \cos \left(\varphi_{g, p}+\frac{\theta_{t}}{2}\right)}{\left(\frac{1+\omega_{p}}{\omega_{p}}\right)^{2}}-d_{h}^{2} \cos \left(\varphi_{h, p}+\frac{\theta_{t}}{2}\right)\right)\right]} .
$$

To evaluate the ratio $\rho_{h, p} / \rho_{g, p}$, we consider the diagram in Fig. 6, which is a 2-D representation of the considered reflection system. Considering the triangle $\mathrm{SAH}, \mathrm{AH}$ $=L_{h, p} \sin (\Delta \varphi)$. Exploiting the triangle AHB, the pointing displacement at the RIS, $\Delta d_{h}$, can be evaluated as

$$
\Delta d_{h}=\frac{L_{h, p} \sin (\Delta \varphi)}{\cos \left(\Delta \varphi+\varphi_{h, p}\right)} .
$$

At the destination, the light seems to be generated from $\mathrm{S}$ ' (see Fig. 6). From the triangle EFD, $\mathrm{EF}=\left(L_{h, p}+L_{g, p}-\right.$ FD) $\tan (\Delta \varphi)$. Considering the triangle FDE, we obtain FD = $\operatorname{EFtan}\left(\varphi_{g, p}\right)$, leading to $\mathrm{EF}=\left[\left(L_{h, p}+L_{g, p}\right) \tan (\Delta \varphi)\right] /[1+$ $\left.\left(\tan (\Delta \varphi) \tan \left(\varphi_{g, p}\right)\right)\right]$. From the same triangle FDE, we have $\Delta d_{g}=\mathrm{EF} / \cos \left(\varphi_{g, p}\right)$. Therefore, the pointing displacement at the destination, $\Delta d_{g}$, can be evaluated as

$$
\Delta d_{g}=\frac{L_{h, p}\left(\frac{\omega_{p}+1}{\omega_{p}}\right) \tan (\Delta \varphi)}{\cos \left(\varphi_{g, p}\right)\left[1+\tan (\Delta \varphi) \tan \left(\varphi_{g, p}\right)\right]} .
$$

Since the number of rays is the same at the RIS and D, applying the standard variance theory, $\rho_{i}^{2}=\sum^{N}\left(x_{i}-\mu_{i}\right)^{2} /(N-1)$, we obtain the ratio $\rho_{h, p} / \rho_{g, p}$ as

$$
\frac{\rho_{h, p}}{\rho_{g, p}}=\left(\frac{\omega_{p}}{\omega_{p}+1}\right) \frac{\cos ^{2}(\Delta \varphi)\left[1+\tan \left(\varphi_{g, p}\right) \tan (\Delta \varphi)\right]}{\cos \left(\varphi_{h, p}+\Delta \varphi\right)} .
$$

Figure 7 depicts the beam waists at the RIS and D versus 


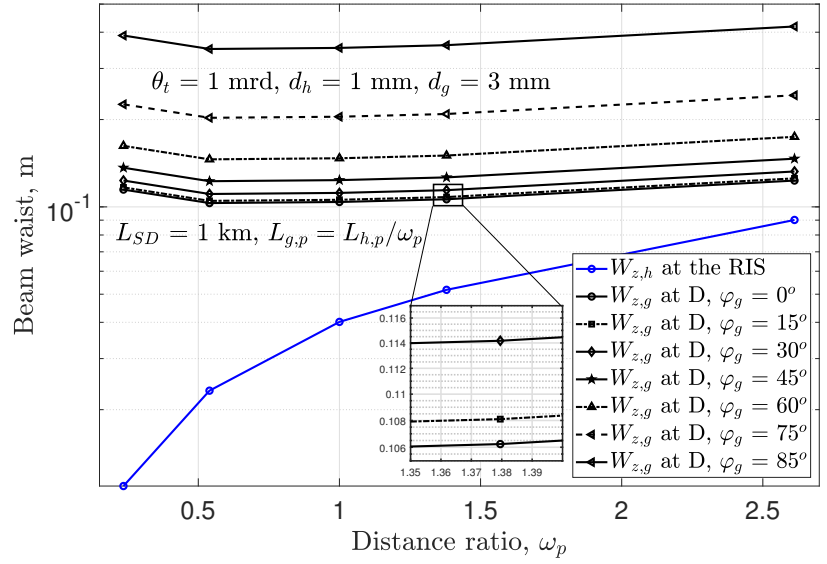

Fig. 7: Bean waist at the RIS and D in terms of incidence angles and PD orientation for selected values of $\omega_{p}$.

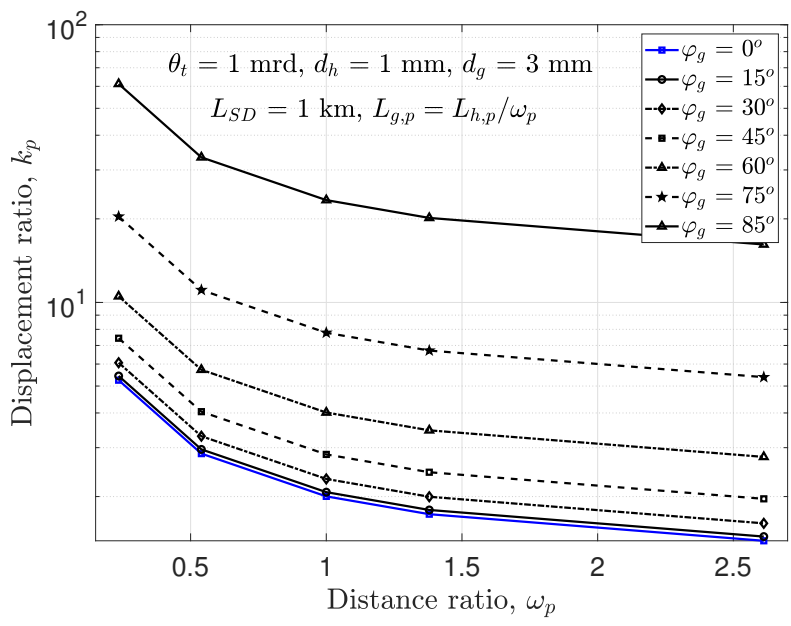

Fig. 8: Ratio of pointing displacement standard deviation at D and the RIS, $k_{p}=\xi_{g, p} / \xi_{h, p}$, versus $\omega_{p}$ for selected values of $\varphi_{h, p}$ and $\varphi_{g, p}$.

$\omega_{p}$ for multiple values of the PD orientation, $\varphi_{g}$. It shows that the beam waist at the RIS increases with $\omega_{p}$, while at D, the beam waist decreases first then increases. However, in all cases, the beam waist at D is greater than that at the RIS. For example, at $\varphi_{g}=0^{\circ}$, we obtain $W_{z, g}=0.115 \mathrm{~m}, 0.103 \mathrm{~m}$, $0.104 \mathrm{~m}, 0.106 \mathrm{~m}$, and $0.123 \mathrm{~m}$, and $W_{z, h}=0.011 \mathrm{~m}, 0.023$ $\mathrm{m}, 0.040 \mathrm{~m}, 0.052 \mathrm{~m}$, and $0.090 \mathrm{~m}$, respectively corresponding to $\mathrm{P}_{3}, \mathrm{P}_{4}, \mathrm{P}_{5}, \mathrm{P}_{6}$, and $\mathrm{P}_{7}\left(\omega_{p}=\{0.235,0.540,1,1.78,2.61\}\right.$, respectively). As Fig. 7 depicts, this pattern is preserved for $\varphi_{g}=\left\{15^{\circ}, 30^{\circ}, 45^{\circ}, 60^{\circ}, 75^{\circ}, 85^{\circ}\right\}$. Figure 8 shows the factor $k_{p}$ versus $\omega_{p}$ at positions $\mathrm{P}_{3}, \mathrm{P}_{4}, \mathrm{P}_{5}, \mathrm{P}_{6}$, and $\mathrm{P}_{7}\left(\omega_{p}=\right.$ $\{0.235,0.540,1,1.78,2.61\})$. As expected, it clearly shows that values obtained for $\varphi_{g}=0^{\circ}$ are always lower than those obtained for $\varphi_{g}>0^{o}$. For example, at $\mathrm{P}_{3}\left(\omega_{p}=0.235\right), k_{p}=$ $5.25,5.44,6.07,7.43,10.52,20.38$, and 61.22 for $\varphi_{g}=0^{\circ}$, $15^{\circ}, 30^{\circ}, 45^{\circ}, 60^{\circ}, 75^{\circ}$, and $85^{\circ}$, respectively. As it can also be seen in Fig. 8, these values of $k_{p}$ decrease as $\omega_{p}$ increases, and indicates how much scintillation disturbs the transmitted signal at D when compared to the disturbance at the RIS for a chosen position of the RIS module and $\varphi_{\mathrm{g}}$.

3) Atmospheric Turbulence: In general, the G-G distribution is used to model channels characterized by moderatedto-strong turbulence levels. Its PDF is given for both subchannels, S-RIS and RIS-D, by [20]

$$
f_{I_{a, i}}\left(I_{a, i}\right)=\frac{2\left(\alpha_{i} \beta_{i}\right)^{\frac{\alpha_{i}+\beta_{i}}{2}}}{\Gamma\left(\alpha_{i}\right) \Gamma\left(\beta_{i}\right)} I_{a, i}^{\frac{\alpha_{i}+\beta_{i}}{2}-1} K_{\alpha_{i}-\beta_{i}}\left(2 \sqrt{\alpha_{i} \beta_{i} I_{a, i}}\right),
$$

where $K_{j}(\cdot)$ is the $j^{t h}$-order modified Bessel function of second kind. The values of $\alpha_{i}$ and $\beta_{i}$ can be calculated using the Rytov variance, $\sigma_{i}^{2}=0.492 C_{i}^{2} \eta^{\frac{7}{6}} L_{i}^{\frac{11}{6}}[24]$, which depends on their altitude-dependent index, $C_{i}^{2}$, which is characterized by the transmission environment, the angular wavenumber, $\eta=\frac{2 \pi}{\lambda}$, and the transmission distance, $L_{i} . \alpha_{i}$ and $\beta_{i}$ are respectively given by $[25$

$$
\alpha_{i}=\left[\exp \left(\frac{0.49 \sigma_{i}^{2}}{\left(1+0.18 x_{i}^{2}+0.56 \sigma_{i}^{12 / 5}\right)^{7 / 6}}\right)-1\right]^{-1},
$$

and

$$
\beta_{i}=\left[\exp \left(\frac{0.51 \sigma_{i}^{2}\left(1+0.69 \sigma_{i}^{12 / 5}\right)^{-5 / 6}}{\left(1+0.9 x_{i}^{2}+0.62 \sigma_{i}^{12 / 5}\right)^{5 / 6}}\right)-1\right]^{-1},
$$

where $x_{i}=\sqrt{\eta d_{i}^{2} / L_{i}}$ and $d_{i}$ the aperture radius.

Rytov Variances, $\sigma_{h}$ and $\sigma_{g}$ : The Rytov variance, which represents the scintillation index of a plane wave in space, is the measure of turbulence power over an optical FSO channel. It is readily demonstrated that the Rytov variance over the RIS-D link, $\sigma_{g}^{2}$, is given by

$$
\sigma_{g}^{2}=\frac{C_{g}^{2}}{C_{h}^{2}} \sigma_{h}^{2} \omega_{p}^{\frac{11}{6}}
$$

where $C_{g}^{2}$ and $C_{h}^{2}$ are respectively the altitude-dependent indices over the RIS-D and S-RIS links, and $\sigma_{h}^{2}$ is the Rytov variance over the S-RIS link. Finally, the G-G turbulence parameters, $\alpha_{g}, \beta_{g}, \alpha_{h}$, and $\beta_{h}$ can be evaluated using Eq. (14), Eq. (15), $x_{h}=\sqrt{\eta d_{h}^{2} / L_{h}}$, and $x_{g}=x_{h} \frac{d_{g}}{d_{h}} \sqrt{\omega_{p}}$.

\section{Performance Analysis}

In this section, we derive unified closed-form expressions for the PDF, CDF, and MGF of the end-to-end SNR.

\section{A. End-to-End Signal-to-Noise Ratio (SNR)}

We assume that the RIS module plays only a reflective function and does not allow light through. We also assume perfect knowledge of the channel phases at the RIS and destination. The detected signal can be expressed as $r=$ $\sqrt{E_{S}}\left(h_{p} \partial e^{j \psi_{p}} g_{p}\right) u+n$, where $E_{S}$ is the symbol energy, $h_{p}$ and $g_{p}$ are respectively the S-RIS and RIS-D complex channel vectors, $\partial e^{j \psi_{p}}$ characterizes the RIS element at the position $p$ with $\partial$ being its amplitude reflection coefficient and $\psi_{p}$ its induced phase [5], [26]. $u$ and $r$ are the transmitted and 
received symbols, respectively, and $n$ is the additive noise at the destination. Note that for the considered RIS structure, the phase-shift profile and matrix given in [12] can be exploited to evaluate $\psi_{p}$ for a fixed orientation of the RIS plane. For any RIS location, the main goal is to adjust the RIS plane orientation such that the reflected light points to the PD's surface and optimizes signal reception at $\mathrm{D}$, which can be achieved through the end-to-end SNR maximization. The SNR is defined by $\gamma=\bar{\gamma}\left|h_{p} \partial e^{j \psi_{p}} g_{p}\right|^{2}$, where $\bar{\gamma}=E_{S} / N_{0}$ represents the end-to-end average SNR, $N_{0}$ is the noise power spectral density at $\mathrm{D}$, and $\psi_{p}$ is a function of the RIS phase-shift matrix.

\section{B. PDF of the End-to-End SNR}

At each location of the RIS, the overall system's gain is given by $h_{p} \partial e^{j \psi_{p}} g_{p}$, where the quantity $\partial e^{j \psi_{p}}$ is deterministic in contrast to $h_{p}$ and $g_{p}$, which are random variables. Thus, the SNR's PDF, $f_{\gamma}(\gamma)$, which can be calculated from the SNRs, $\gamma_{h}$ and $\gamma_{g}$, can be evaluated as [27, Eq. (5)]

$$
f_{\gamma}(\gamma)=\int_{0}^{\infty} f_{\gamma_{h}}(t) f_{\gamma_{g}}\left(\frac{\gamma}{t}\right) \frac{1}{t} d t
$$

where $f_{\gamma_{h}}(\cdot)$ and $f_{\gamma_{g}}(\cdot)$ are respectively the PDFs of the S-RIS and RIS-D sub-channel's SNRs, $\gamma_{h}$ and $\gamma_{g}$. With the assumption of a constant weather condition over the environment, both parts of the channel can be modeled by a combined distribution including pointing errors and turbulence levels [1, Eq. (10)]. The two sub-channels being characterized by $\alpha_{h}, \beta_{h}$, and $\xi_{h}$ for the S-RIS link, and $\alpha_{g}, \beta_{g}$, and $\xi_{g}$ for the S-RIS link, their PDF can be expressed as [1, Eq. (10)]

$$
f_{\gamma_{i}}\left(\gamma_{i}\right)=\frac{M_{i}}{\gamma_{i}} \mathbf{G}_{1,3}^{3,0}\left[Q_{i}\left(\frac{\gamma_{i}}{\bar{\gamma}_{i}}\right)^{\frac{1}{a}} \mid \begin{array}{c}
\xi_{i}^{2}+1 \\
\xi_{i}^{2}, \alpha_{i}, \beta_{i}
\end{array}\right]
$$

where $i \in\{h, g\}, M_{i}=\xi_{i}^{2} / a \Gamma\left[\alpha_{i}\right] \Gamma\left[\beta_{i}\right], Q_{i}=\xi_{i}^{2} \alpha_{i} \beta_{i} /\left(\xi_{i}^{2}+\right.$ 1), $a \in\{1,2\}$ indicates whether the transmission utilizes the heterodyne (HD) $(a=1)$ or the intensity modulation/direct detection (IM/DD) $(a=2)$ techniques $|1|$, and $\mathbf{G}_{p, q}^{m, n}\left[z \mid \begin{array}{l}a_{p} \\ b_{q}\end{array}\right]$ is the Meijer-G function. We sequentially substitute $\gamma_{i}$ by $t$ and $\frac{\gamma}{t}$ in (18), and obtain $f_{\gamma_{h}}(t)$ and $f_{\gamma_{g}}\left(\frac{\gamma}{t}\right)$ respectively as

$$
f_{\gamma_{h}}(t)=\frac{M_{h}}{t} \mathbf{G}_{1,3}^{3,0}\left[Q_{h}\left(\frac{t}{\bar{\gamma}_{h}}\right)^{\frac{1}{a}} \mid \begin{array}{c}
\xi_{h}^{2}+1 \\
\xi_{h}^{2}, \alpha_{h}, \beta_{h}
\end{array}\right],
$$

and

$$
f_{\gamma_{g}}\left(\frac{\gamma}{t}\right)=\frac{M_{g} t}{\gamma} \mathbf{G}_{1,3}^{3,0}\left[Q_{g}\left(\frac{\gamma}{\bar{\gamma}_{g} t}\right)^{\frac{1}{a}} \mid \begin{array}{c}
\xi^{2}+1 \\
\xi_{g}^{2}, \alpha_{g}, \beta_{g}
\end{array}\right],
$$

where $\bar{\gamma}_{h}$ and $\bar{\gamma}_{g}$ are average values of the SNRs $\gamma_{h}$ and $\gamma_{g}$, respectively. In 20], the variable $t$ appears at the denominator. To obtain a Meijer-G function with a numerator-based variable $t$, we apply the reflection property of the Meijer-G function, given by [28]

$$
\mathbf{G}_{p, q}^{m, n}\left[z \mid \begin{array}{l}
A_{p} \\
B_{q}
\end{array}\right]=\mathbf{G}_{q, p}^{n, m}\left[z^{-1} \mid \begin{array}{l}
1-B_{q} \\
1-A_{p}
\end{array}\right]
$$

to 20, and obtain

$$
f_{\gamma_{g}}\left(\frac{\gamma}{t}\right)=\frac{M_{g} t}{\gamma} \mathbf{G}_{3,1}^{0,3}\left[\frac{1}{Q_{g}}\left(\frac{\bar{\gamma}_{g}}{\gamma}\right)^{\frac{1}{a}} t^{\frac{1}{a}} \mid \begin{array}{c}
1-\xi_{g}^{2}, 1-\alpha_{g}, 1-\beta_{g} \\
-\xi_{g}^{2}
\end{array}\right] .
$$

To get the end-to-end SNR's PDF, $f_{\gamma}(\gamma)$, we substitute Eqs. (19) and (22) into (17), which leads to

$$
\begin{aligned}
f_{\gamma}(\gamma) & =\frac{M_{g} M_{h}}{\gamma} \int_{0}^{\infty} \frac{1}{t} \mathbf{G}_{1,3}^{3,0}\left[Q_{h}\left(\frac{t}{\bar{\gamma}_{h}}\right)^{\frac{1}{a}} \mid \begin{array}{c}
\xi_{h}^{2}+1 \\
\xi_{h}^{2}, \alpha_{h}, \beta_{h}
\end{array}\right] \\
& \times \mathbf{G}_{3,1}^{0,3}\left[\frac{1}{Q_{g}}\left(\frac{\bar{\gamma}_{g}}{\gamma}\right)^{\frac{1}{a}} t^{\frac{1}{a}}\left[\begin{array}{c}
1-\xi_{g}^{2}, 1-\alpha_{g}, 1-\beta_{g} \\
-\xi_{g}^{2}
\end{array}\right] d \gamma .\right.
\end{aligned}
$$

Applying the change of variable $X=t^{\frac{1}{a}} \Rightarrow t=X^{a}$, and $d t=a X^{a-1} d X$, we obtain

$$
\begin{aligned}
f_{\gamma}(\gamma) & =\frac{a M_{g} M_{h}}{\gamma} \int_{0}^{\infty} \frac{1}{X} \mathbf{G}_{1,3}^{3,0}\left[\frac{Q_{h} X}{\bar{\gamma}_{h}^{\frac{1}{a}}} \mid \begin{array}{c}
\xi_{h}^{2}+1 \\
\xi_{h}^{2}, \alpha_{h}, \beta_{h}
\end{array}\right] \\
& \times \mathbf{G}_{3,1}^{0,3}\left[\frac{1}{Q_{g}}\left(\frac{\bar{\gamma}_{g}}{\gamma}\right)^{\frac{1}{a}} X \mid \begin{array}{c}
1-\xi_{g}^{2}, 1-\alpha_{g}, 1-\beta_{g} \\
-\xi_{g}^{2}
\end{array}\right] d X
\end{aligned}
$$

With the help of [29. Eq. (07.34.21.0011.01)], we evaluate the integral in 24, apply the identity in 21, , and obtain the exact unified PDF of end-to-end SNR, $f_{\gamma}(\gamma)$, as

$f_{\gamma}(\gamma)=\frac{a M_{h} M_{g}}{\gamma} \mathbf{G}_{2,6}^{6,0}\left[Q_{h} Q_{g}\left(\frac{\gamma}{\bar{\gamma}_{\mathrm{eq}}}\right)^{\frac{1}{a}} \mid \begin{array}{c}\xi_{h}^{2}+1, \xi_{g}^{2}+1 \\ \xi_{g}^{2}, \alpha_{g}, \beta_{g}, \xi_{h}^{2}, \alpha_{h}, \beta_{h}\end{array}\right]$,

where $\bar{\gamma}_{\mathrm{eq}}=\bar{\gamma}_{g} \bar{\gamma}_{h}$

\section{CDF of the End-to-End SNR}

The CDF of the end-to-end SNR, $F_{\gamma}(\gamma)$, can be calculated as $F_{\gamma}(\gamma)=\int_{0}^{\infty} f_{\gamma}(\gamma) d \gamma$. Substituting the expression of $f_{\gamma}(\gamma)$ Eq. 25, and permuting the variables $\gamma, x$, and $\infty$, we obtain

$$
\begin{aligned}
F_{\gamma}(\gamma) & =a M_{h} M_{g} \int_{0}^{\gamma} \frac{1}{x} \\
& \times \mathbf{G}_{2,6}^{6,0}\left[Q_{h} Q_{g}\left(\frac{\gamma}{\bar{\gamma}_{\mathrm{eq}}}\right)^{\frac{1}{a}} \mid \begin{array}{c}
\xi_{h}^{2}+1, \xi_{g}^{2}+1 \\
\xi_{g}^{2}, \alpha_{g}, \beta_{g}, \xi_{h}^{2}, \alpha_{h}, \beta_{h}
\end{array}\right] d x .
\end{aligned}
$$

With the help of [29, Eq. (07.34.21.0084.01)], we evaluate the integral in 26, to get the closed-form expression of $F_{\gamma}(\gamma)$ as

$$
F_{\gamma}(\gamma)=M_{\mathrm{eq}} \mathbf{G}_{2 a+1,6 a+1}^{6 a, 1}\left[Q_{\mathrm{eq}}\left(\frac{\gamma}{\bar{\gamma}_{\mathrm{eq}}}\right) \mid \begin{array}{c}
1, \Delta_{1} \\
\Delta_{2}, 0
\end{array}\right],
$$

where $M_{\text {eq }}=\frac{M_{h} M_{g} a^{\alpha} h^{+\alpha_{g}+\beta_{h}+\beta_{g}-2}}{(2 \pi)^{2(a-1)}}, Q_{\text {eq }}=\frac{\left(Q_{h} Q_{g}\right)^{a}}{a^{4 a}}, \Delta_{1}=\frac{\xi_{h}^{2}+1}{a}$, $\ldots, \frac{\xi_{h}^{2}+a}{a}, \frac{\xi_{g}^{2}+1}{a}, \ldots, \frac{\xi_{g}^{2}+a}{a}$ with $2 a$ terms, and $\Delta_{2}=\frac{\xi_{g}^{2}}{a}, \ldots$, $\frac{\xi_{g}^{2}+a-1}{a}, \frac{\alpha_{g}}{a}, \ldots, \frac{\alpha_{g}+a-1}{a}, \frac{\beta_{g}}{a}, \ldots, \frac{\beta_{g}+a-1}{a}, \frac{\xi_{h}^{2}}{a}, \ldots, \frac{\xi_{h}^{2}+a-1}{a}$, $\frac{\alpha_{h}}{a}, \ldots, \frac{\alpha_{h}+a-1}{a}, \frac{\beta_{h}}{a}, \ldots, \frac{\beta_{h}+a-1}{a}$ with $6 a$ terms. 


\section{Moment Generating Function (MGF)}

The MGF, $\Omega_{\gamma}(s)$, is readily calculated from the CDF as [1. Eq. (15)]

$$
\Omega_{\gamma}(s)=s \int_{0}^{\infty} \exp (-\gamma s) F_{\gamma}(\gamma) d \gamma .
$$

Substituting 27] into (28), we obtain

$$
\Omega_{\gamma}(s)=M_{\mathrm{eq}} s \int_{0}^{\infty} e^{-\gamma s} \mathbf{G}_{2 a+1,6 a+1}^{6 a, 1}\left[Q_{\mathrm{eq}}\left(\frac{\gamma}{\bar{\gamma}_{\mathrm{eq}}}\right) \mid \begin{array}{c}
1, \Delta_{1} \\
\Delta_{2}, 0
\end{array}\right] d \gamma .
$$

Using [30, Eq. (7.813.1)], we evaluate the integral in (29) and obtain the closed-form and unified expression of the MGF as

$$
\Omega_{\gamma}(s)=M_{\mathrm{eq}} \mathbf{G}_{2 a+2,6 a+1}^{6 a, 2}\left[\frac{Q_{\mathrm{eq}}}{\overline{\bar{\gamma}}_{\mathrm{eq}} s} \mid \begin{array}{c}
0,1, \Delta_{1} \\
\Delta_{2}, 0
\end{array}\right] .
$$

\section{Applications}

In this section, we analyze the performance of the proposed RIS-aided T-FSO system in terms of the OP, $P_{\text {out }}$, ergodic channel capacity, $\bar{C}$, and average BER, $\bar{P}_{b}$, for selected binary schemes, considering multiple RIS positions $\left(\omega_{p}\right)$, and PD orientation $\left(\varphi_{g, p}\right)$, and the ratio $k_{p}$.

\section{A. Outage Probability}

Outage occurs when the end-to-end SNR, $\gamma$, falls below a threshold value, $\gamma_{t h}=e^{2 R-1}$, predefined for a specific qualityof-service, $R$ being the transmission rate. This implies that under such conditions, the system does not reach the specific rate $R$. The OP, $P_{\text {out }}$, which defines this failure, can be readily calculated from (27) by finding $F_{\gamma}\left(\gamma_{t h}\right)$.

\section{B. Ergodic Channel Capacity}

In the proposed system, the channel state information is not available at the transmitter, and data is transmitted without instantaneous feedback, which reduces the system capacity [1]. The transmitted symbol is long enough so that data is encoded over all the possible channel fading states, and the atmospheric turbulence. In T-FSO, this channel is slowfading and remains constant over the symbol transmission. The atmospheric turbulence is combined with the effects of pointing errors and make the signal fluctuate at a very high rate [1]. Thus, the overall channel statistical properties can be evaluated during the transmission of a single symbol. Therefore, the ergodic channel analysis can be realized [1], 31]. The ergodic channel capacity, $\bar{C}$, is given by

$$
\bar{C}=\frac{1}{\ln (2)} \int_{0}^{\infty} \ln (1+\chi \gamma) f_{\gamma}(\gamma) d \gamma,
$$

where $\chi=1$ for HD and $\chi=\frac{e}{2 \pi}$ for IM/DD [1]. Exploiting the Meijer's G-function representation of $\ln (1+x)$ [29, Eq. (07.34.03.0456.01)] and substituting [25) in (31), $\bar{C}$ becomes

$$
\begin{aligned}
\bar{C} & =\frac{a M_{h} M_{g}}{\ln (2)} \int_{0}^{\infty} \frac{1}{\gamma} \mathbf{G}_{2,2}^{1,2}\left[\chi \gamma \mid \begin{array}{l}
1,1 \\
1,0
\end{array}\right] \\
& \times \mathbf{G}_{2,6}^{6,0}\left[Q_{h} Q_{g}\left(\frac{\gamma}{\bar{\gamma}_{\mathrm{eq}}}\right)^{\frac{1}{a}} \mid \begin{array}{c}
\xi_{h}^{2}+1, \xi_{g}^{2}+1 \\
\xi_{g}^{2}, \alpha_{g}, \beta_{g}, \xi_{h}^{2}, \alpha_{h}, \beta_{h}
\end{array}\right] d \gamma .
\end{aligned}
$$

With the help of [29. Eq. (07.34.21.0013.01)], we evaluate the integral in 32 and obtain a closed-form unified expression of $\bar{C}$ as

$$
\bar{C}=\frac{M_{\mathrm{eq}}}{\ln (2)} \mathbf{G}_{2 a+2,6 a+2}^{6 a+2,1}\left[\frac{Q_{\mathrm{eq}}}{\chi \bar{\gamma}_{\mathrm{eq}}} \mid \begin{array}{c}
0,1, \Delta_{1} \\
\Delta_{2}, 0,0
\end{array}\right] .
$$

\section{Average Bit Error Rate (BER) for Selected Binary Schemes}

In data transmission, the BER is a classical metric used to evaluate the system performance. Considering that in the proposed system, binary schemes are used to modulate the data before transmission, the average BER, $\bar{P}_{b}$, can be evaluated using [32. Eq. (13)]

$$
\bar{P}_{b}=\frac{q^{p}}{2 \Gamma(p)} \int_{0}^{\infty} e^{-q \gamma} \gamma^{p-1} F_{\gamma}(\gamma) d \gamma
$$

where the pair $(p, q)$ defines the binary modulation schemes [1]. The values of $p$ and $q$ for selected modulation schemes, namely, CBFSK, NBFSK, CBPSK, and DBPSK, are respectively given by the pairs $(0.5,0.5),(1,0.5),(0.5,1)$, and $(1$, 1). Substituting (27) into (34) leads to

$$
\bar{P}_{b}=\frac{q^{p} M_{\mathrm{eq}}}{2 \Gamma(p)} \int_{0}^{\infty} \frac{e^{-q \gamma}}{\gamma^{1-p}} \mathbf{G}_{2 a+1,6 a+1}^{6 a, 1}\left[Q_{\mathrm{eq}}\left(\frac{\gamma}{\bar{\gamma}_{\mathrm{eq}}}\right) \mid \begin{array}{c}
1, \Delta_{1} \\
\Delta_{2}, 0
\end{array}\right] d \gamma .
$$

Using [30, Eq. (7.813.1)], the integral in (35) can be evaluated to obtain a closed-form unified expression of the average BER, $\bar{P}_{b}$, as

$$
\bar{P}_{b}=\frac{M_{\mathrm{eq}}}{2 \Gamma(p)} \mathbf{G}_{2 a+2,6 a+1}^{6 a, 2}\left[\frac{Q_{\mathrm{eq}}}{q \bar{\gamma}_{\mathrm{eq}}} \mid \begin{array}{c}
1-p, 1, \Delta_{1} \\
\Delta_{2}, 0
\end{array}\right] .
$$

\section{Diversity Order and Coding Gain}

The diversity order defines the increase in SNR due to some diversity schemes. Practically, it determines the slope of the $\bar{P}_{b}=f(\gamma)$ curve. On the other hand, the coding gain is the difference between the SNR levels for coded and un-coded systems, required to reach the same $\bar{P}_{b}$. At high SNR, the average BER, $\bar{P}_{b}$, can be approximated as $\bar{P}_{b}=\left(G_{c} \bar{\gamma}\right)^{-G d}[33]$. We use the Meijer-G function expansion [29. Eq. (07.34.06.0017.01)], associated with the unity of $\lim _{x \rightarrow \infty}{ }_{c} F_{d}[e ; f ; x]$ [1], [34], to find the unified asymptotic expression of $\bar{P}_{b}$, as

$$
\bar{P}_{b} \approx \frac{M_{\mathrm{eq}}}{2 \Gamma(p)} \sum_{k=1}^{6 a} \xi(i, j, k)\left[\frac{q \bar{\gamma}_{\mathrm{eq}}}{Q_{\mathrm{eq}}}\right]^{-\left(\Delta_{2, k}\right)},
$$

where $\xi(i, j, k)$ is expressed as

$$
\xi(i, j, k)=\frac{\Gamma\left(\Delta_{2, k}+p\right) \prod_{j=1 ; j \neq k}^{6 a} \Gamma\left(\Delta_{2, j}-\Delta_{2, k}\right)}{\Delta_{2, k} \prod_{i=3}^{2 a+2} \Gamma\left(\Delta_{1, i}-\Delta_{2, k}\right)},
$$

where $\Delta_{1, i}=\Delta_{1,1}, \Delta_{1,2}, \ldots, \Delta_{1,2 a+2}$ with $2 a+2$ terms, $\Delta_{2, j}=$ $\Delta_{2,1}, \Delta_{2,2}, \ldots, \Delta_{2,6 a}$ with $6 a$ terms, and $\Delta_{2, k}=\Delta_{2,1}, \Delta_{2,2}, \ldots$, $\Delta_{2,6 a}$ with $6 a$ terms. By comparing (37) to $\bar{P}_{b}=\left(G_{c} \bar{\gamma}\right)^{-G d}$, we obtain the unified expressions of the diversity order and the coding gain as $G_{d}=\min \left(\frac{\xi_{g}^{2}}{a}, \frac{\alpha_{g}}{a}, \frac{\beta_{g}}{a}, \frac{\xi_{h}^{2}}{a}, \frac{\alpha_{h}}{a}, \frac{\beta_{h}}{a}\right)$ and

$$
G_{c}=\frac{q}{Q_{\mathrm{eq}}}\left[\sum_{k=1}^{6 a} \xi(i, j, k) \frac{M_{\mathrm{eq}}}{2 \Gamma(p)}\right]^{\frac{-1}{\mathrm{\Lambda}_{2, k}}},
$$


TABLE I: System parameters.

\begin{tabular}{|c|c|c|c|c|c|c|c|c|c|}
\hline \multicolumn{10}{|c|}{$\begin{array}{c}y_{S}=0.25 \mathrm{~km}, \phi=8.1^{\circ}, L_{S D}=1 \mathrm{~km}, x_{K}=9.98 \mathrm{~km}, \xi_{h}=1.1, C_{h}^{2}=C_{g}^{2}=2 \times 10^{-13} \mathrm{~m}^{-2 / 3}, \\
\lambda=10^{3} \mathrm{~nm}, d_{h}=1 \mathrm{~mm}, d_{g}=3 \mathrm{~mm}, \theta_{t}=0.175 \mathrm{mrd}, \Delta \varphi=1 \mathrm{mrd}, \text { and } \lambda=1000 \mathrm{~nm}\end{array}$} \\
\hline Positions & $\overline{\mathrm{P}_{1}}$ & $\overline{\mathrm{P}_{2}}$ & $\mathrm{P}_{3}$ & $\mathrm{P}_{4}$ & $\mathrm{P}_{5}\left(L_{h}=L_{g}\right)$ & $\mathrm{P}_{6}$ & $\mathrm{P}_{7}$ & $\mathrm{P}_{8}$ & $\mathrm{P}_{9}$ \\
\hline$x_{p}(\mathrm{~km})$ & -0.66 & -0.33 & 0 & 0.33 & 0.54 & 0.66 & 0.99 & 1.32 & 1.65 \\
\hline$\varphi_{h, p}$ & $-69.25^{\circ}$ & $-52.85^{\circ}$ & $0^{\circ}$ & $52.85^{\circ}$ & $65.18^{\circ}$ & $69.25^{\circ}$ & $75.83^{\circ}$ & $79.28^{\circ}$ & $81.38^{\circ}$ \\
\hline$\varphi_{g, p}$ & $76.67^{\circ}$ & $73.50^{\circ}$ & $68.45^{\circ}$ & $59.36^{\circ}$ & $41.02^{\circ}$ & $40.17^{\circ}$ & $0^{o}$ & $-40.17^{\circ}$ & $-59.36^{\circ}$ \\
\hline$L_{h, p}(\mathrm{~km})$ & 0.71 & 0.41 & 0.25 & 0.41 & 0.60 & 0.71 & 1.02 & 1.34 & 1.67 \\
\hline$L_{g, p}(\mathrm{~km})$ & 1.70 & 1.38 & 1.06 & 0.77 & 0.60 & 0.51 & 0.39 & 0.51 & 0.77 \\
\hline$\omega_{p}$ & 0.42 & 0.30 & 0.24 & 0.54 & 1.00 & 1.38 & 2.61 & 2.63 & 2.18 \\
\hline$\alpha_{h, p}$ & 2.49 & 4.59 & 10.26 & 4.59 & 2.91 & 2.49 & 2.09 & 2.09 & 2.24 \\
\hline$\beta_{h, p}$ & 3.90 & 5.31 & 10.55 & 5.31 & 4.04 & 3.90 & 4.49 & 5.89 & 7.84 \\
\hline$\alpha_{g, p}$ & 7.83 & 35.37 & 139.41 & 12.48 & 2.92 & 2.11 & 2.91 & 3.54 & 3.62 \\
\hline$\beta_{g, p}$ & 8.27 & 34.65 & 135.25 & 12.74 & 4.08 & 4.37 & 16.48 & 26.70 & 28.07 \\
\hline \multicolumn{10}{|c|}{$\varphi_{g}=0^{o}$} \\
\hline$k_{p}$ & 3.40 & 4.32 & 5.25 & 2.86 & 2.00 & 1.73 & 1.39 & 1.38 & 1.46 \\
\hline$\xi_{g, p}$ & 3.74 & 4.75 & 5.78 & 3.14 & 2.20 & 1.90 & 1.52 & 1.52 & 1.61 \\
\hline \multicolumn{10}{|c|}{$\varphi_{g}=15^{\circ}$} \\
\hline$k_{p}$ & 3.52 & 4.48 & 5.44 & 2.96 & 2.07 & 1.79 & 1.44 & 1.43 & 1.52 \\
\hline$\xi_{g, p}$ & 3.87 & 4.92 & 5.98 & 3.25 & 2.28 & 1.97 & 1.58 & 1.58 & 1.67 \\
\hline \multicolumn{10}{|c|}{$\varphi_{g}=30^{\circ}$} \\
\hline$k_{p}$ & 3.93 & 4.99 & 6.07 & 3.30 & 2.31 & 2.00 & 1.60 & 1.60 & 1.69 \\
\hline$\xi_{g, p}$ & 4.32 & 5.49 & 6.67 & 3.63 & 2.55 & 2.20 & 1.76 & 1.76 & 1.86 \\
\hline \multicolumn{10}{|c|}{$\varphi_{g}=45^{\circ}$} \\
\hline$k_{p}$ & 4.81 & 6.12 & 7.43 & 4.04 & 2.84 & 2.45 & 1.96 & 1.96 & 2.07 \\
\hline$\xi_{g, p}$ & 5.29 & 6.73 & 8.18 & 4.45 & 3.12 & 2.69 & 2.16 & 2.16 & 2.28 \\
\hline \multicolumn{10}{|c|}{$\varphi_{g}=60^{\circ}$} \\
\hline$k_{p}$ & 6.81 & 8.66 & 10.52 & 5.72 & 4.01 & 3.46 & 2.78 & 2.78 & 2.94 \\
\hline$\xi_{g, p}$ & 7.49 & 9.53 & 11.57 & 6.30 & 4.42 & 3.81 & 3.06 & 3.05 & 3.23 \\
\hline \multicolumn{10}{|c|}{$\varphi_{g}=75^{\circ}$} \\
\hline$k_{p}$ & 13.20 & 16.78 & 20.38 & 11.09 & 7.78 & 6.71 & 5.38 & 5.38 & 5.69 \\
\hline$\xi_{g, p}$ & 14.52 & 18.46 & 22.42 & 12.20 & 8.56 & 7.38 & 5.92 & 5.92 & 6.26 \\
\hline \multicolumn{10}{|c|}{$\varphi_{g}=85^{\circ}$} \\
\hline$k_{p}$ & 39.65 & 50.42 & 61.22 & 33.30 & 23.36 & 20.15 & 16.17 & 16.16 & 17.09 \\
\hline$\xi g, p$ & 43.62 & 55.46 & 67.34 & 36.63 & 25.70 & 22.17 & 17.79 & 17.78 & 18.80 \\
\hline
\end{tabular}

respectively.

\section{RESULTS}

We consider a T-FSO transmission environment in which D is situated at $L_{S D}=1 \mathrm{~km}$ from $\mathrm{S}$. We analyze the proposed system for 9 different positions of the RIS module, $\mathrm{P}_{1}$ to $\mathrm{P}_{9}$, as shown in Fig. 2. The corresponding coordinates are $(-0.66$, $0),(-0.33,0),(0,0),(0.33,0),(0.54,0),(0.66,0),(0.99,0)$, $(1.32,0),(1.65,0)$ (in $\mathrm{km})$, respectively. The corresponding incidence angles and S-RIS link lengths are $\varphi_{h, p}=\left\{-69.25^{\circ}\right.$, $\left.-52.85^{\circ}, 0^{\circ}, 52.85^{\circ}, 65.18^{\circ}, 69.25^{\circ}, 75.83^{\circ}, 79.28^{\circ}, 81.38^{\circ}\right\}$, $L_{h, p}=\{0.71,0.41,0.25,0.41,0.60,0.71,1.02,1.34,1.67\}$,

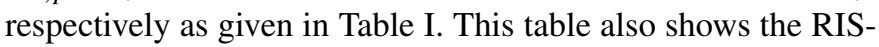
D distances, $L_{g, p}$, and ratios, $\omega_{p}$, related to the 9 positions of the RIS module. A combination of these values lead to $k_{p}$, also given in Table I for selected PD's orientations, $\varphi_{g, p}$. The chosen positions of $S(0,0.250)$ and $\mathrm{D}(0.99,0.391)$, combined with the misalignment coefficients, $\xi_{h, p}=1.1$, and the subchannels refractive structures and indices $C_{h}^{2}=C_{g}^{2}=2 \times 10^{-13}$ $\mathrm{m}^{-2 / 3}$, are exploited to calculate the atmospheric turbulence parameters, $\alpha_{h, p}, \alpha_{g, p}, \beta_{h, p}$, and $\beta_{g, p}$, and the misalignment coefficients $\xi_{g, p}$. These parameters and coefficients vary with the RIS position and PD orientation, as given in Table I.

In this performance analysis of RIS-based T-FSO systems, we consider the most used system performance evaluations metrics, which are the OP, $P_{\text {out }}$, channel capacity, $\bar{C}$, and error probability, $\bar{P}_{b}$. We exploit the channel and system parameters given in Table II to plot $F_{\gamma}\left(\gamma_{t h}\right)$ against $\bar{\gamma}_{\mathrm{eq}} / \gamma_{t h}$ for the 9 positions of the RIS module, and $\varphi_{g}=0^{o} . F_{\gamma}\left(\gamma_{t h}\right)$ is also plotted against selected values of $\varphi_{g}$ for two positions of the RIS module, and finally, $F_{\gamma}\left(\gamma_{t h}\right)$ is plotted versus $\omega_{p}$ for $\varphi_{g}=0^{\circ}$. On the other hand, the channel capacity, $\bar{C}$, and error probability, $\bar{P}$, are given against $\bar{\gamma}_{\mathrm{eq}}$ and $\omega_{p}$, for the same selected system's parameters. Finally, we discuss the asymptotic behavior of the BER curves to highlight the diversity order.

The two system's sub-channels refractive structure and indices, $C_{h}^{2}$ and $C_{g}^{2}$, which may be similar or different, remain constant during the transmission of one symbol. Their values define how moderate or strong is the atmospheric turbulence over the sub-channels. For such scenarios, their values range from $10^{-14}$ to $10^{-9}$, where $10^{-9}$ represents the strongest turbulence levels [35]. We consider that $C_{h}^{2}=C_{g}^{2}=2 \times 10^{-13}$ $\mathrm{m}^{-2 / 3}$.

Using Eqs. (14), (15), and the analysis provided in Section II. we obtain the values of $\alpha_{h, p}, \beta_{h, p}, \xi_{h, p}, \alpha_{g, p}, \beta_{g, p}, \xi_{g, p}$, and $k_{p}$, corresponding to positions $\mathrm{P}_{1}, \mathrm{P}_{2}, \mathrm{P}_{3}, \mathrm{P}_{4}, \mathrm{P}_{5}, \mathrm{P}_{6}$, $\mathrm{P}_{7}, \mathrm{P}_{8}$, and $\mathrm{P}_{9}, \varphi_{g}=0^{\circ}, 15^{\circ}, 30^{\circ}, 45^{\circ}, 60^{\circ}, 75^{\circ}$, and $85^{\circ}$, which are given in Table II These values are calculated for the RIS and receiver apertures of $d_{h}=3 \mathrm{~mm}$ and $d_{g}=1 \mathrm{~mm}$, respectively, and a wavelength, $\lambda=1000 \mathrm{~nm}$.

First, we analyze the OP, $P_{\text {out }}$, then the average channel capacity, $\bar{C}$, and the average BER, $\bar{P}_{b}$ with fixed effect of the pointing error, $\xi_{h}=1.1$. Figures 9 , 10, and 11 depict the OP against the normalized electrical SNR and ratio $\omega_{p}$. Figure 9 describes the probability of failure of the RIS-aided 


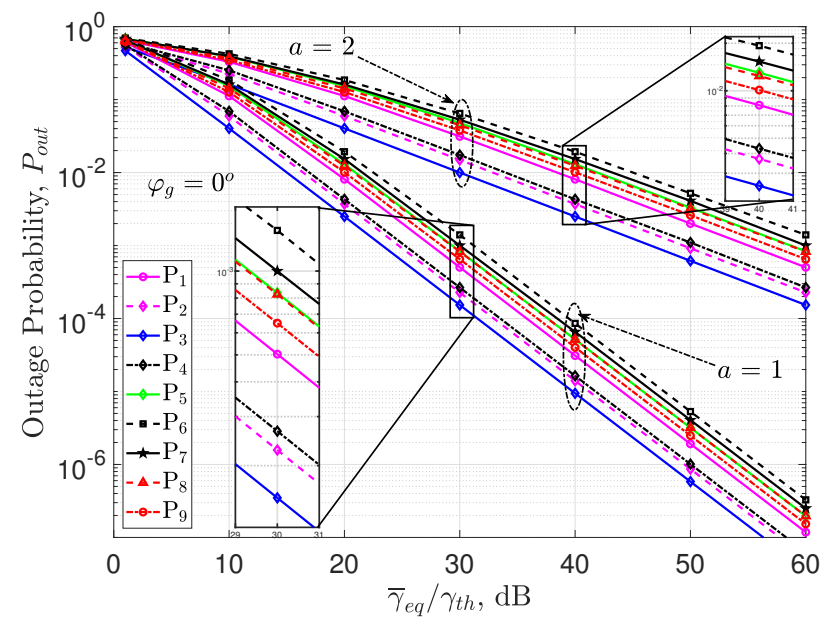

Fig. 9: Outage probability, $P_{\text {out }}$, versus $\bar{\gamma}_{\mathrm{eq}} / \gamma_{t h}$ for several positions of the RIS module, when $\varphi_{g}=10^{\circ}, a=\{1,2\}$, and $\xi_{h, p}=1.1$.

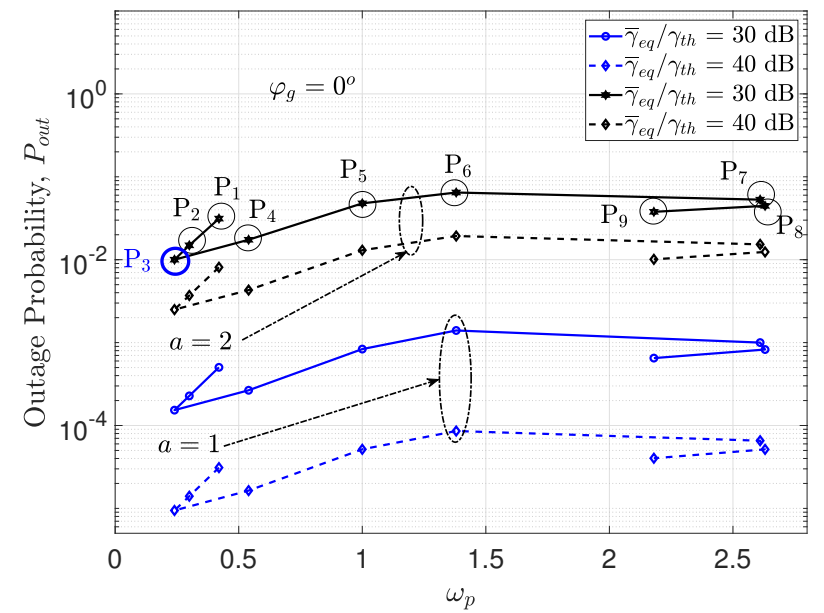

Fig. 10: Outage probability, $P_{\text {out }}$, versus $\omega_{p}$ for $\varphi_{g}=0^{o}$, $\bar{\gamma}_{\mathrm{eq}} / \gamma_{t h}=30 \mathrm{~dB}, 40 \mathrm{~dB}, a=\{1,2\}$, and $\xi_{h, p}=1.1$.

T-FSO system in terms of the RIS position $\left(\omega_{p}\right)$ for both the HD and IM/DD techniques, while Fig. 11 focuses on the impact of $\omega_{p}$, and Fig. 10 highlights the impact of $\varphi_{g, p}$. Similarly, Fig. 12 shows the channel capacity in terms of the different RIS positions for the HD and IM/DD techniques, while Figs. 14 and 10 respectively give the channel capacity against $\bar{\gamma}_{\mathrm{eq}}$, showing the impact of the RIS position and the PD orientation. Figures 16 and 18 depict BER results for DBPSK versus $\bar{\gamma}_{\mathrm{eq}}$ for selected positions of the RIS module (Fig. 16, and $\omega_{p}$ (Fig. 18). Lastly, Figs. 19 and 19 respectively compare the selected schemes for several values of $\varphi_{g, p}$, and selected binary schemes. To validate the accuracy of our derivations, both numerical and simulated results are presented.

Considering the proposed model in Fig. 2, we analysis $P_{\text {out }}$, $\bar{C}$, and $\bar{P}_{b}$, taking into account the 3 portions of the figure: $(i)$ $\left\{\mathrm{P}_{1}, \mathrm{P}_{2}, \mathrm{P}_{3}\right\}$, (ii) $\left\{\mathrm{P}_{3}, \mathrm{P}_{4}, \mathrm{P}_{5}, \mathrm{P}_{6}, \mathrm{P}_{7}\right\}$, and (iii) $\left\{\mathrm{P}_{7}, \mathrm{P}_{8}, \mathrm{P}_{9}\right\}$.

\section{- Outage Probability}

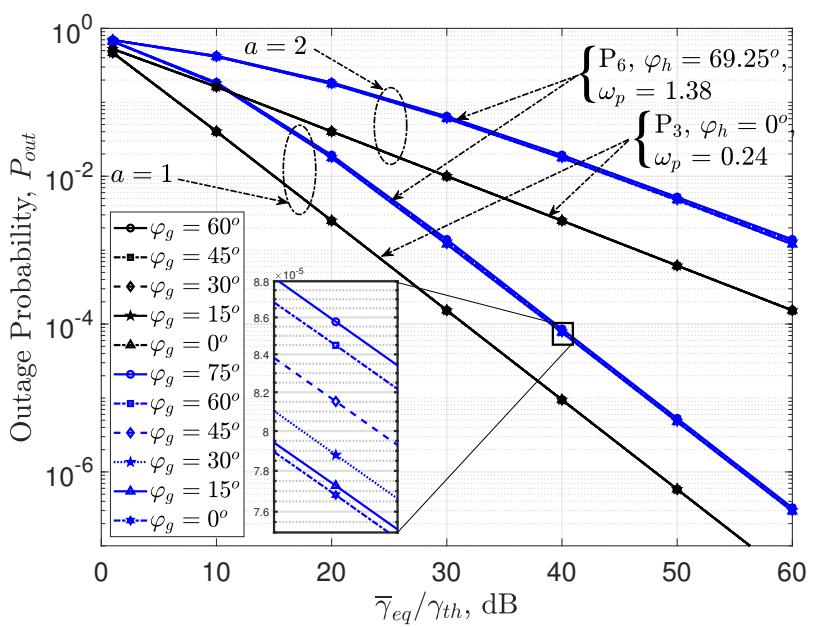

Fig. 11: Outage probability, $P_{\text {out }}$, versus $\bar{\gamma}_{\mathrm{eq}} / \gamma_{t h}$ for $\varphi_{g}=\left\{0^{\circ}\right.$, $\left.15^{\circ}, 30^{\circ}, 45^{\circ}, 60^{\circ}, 75^{\circ}\right\}, \mathrm{P}_{3}$ and $\mathrm{P}_{6}, a=\{1,2\}$, and $\xi_{h, p}=$ 1.1 .

(i) $-\left\{\mathrm{P}_{1}, \mathrm{P}_{2}, \mathrm{P}_{3}\right\}$. Figure 9 shows that as one moves from $\mathrm{P}_{3}$ to $\mathrm{P}_{1}$, the probability of failure of the system increases for both HD and IM/DD techniques. This result is confirmed by Fig. 10, where $P_{\text {out }}$ is given versus $\omega_{p}$ for $\bar{\gamma}_{\mathrm{eq}} / \gamma_{t h} 30 \mathrm{~dB}$ and $40 \mathrm{~dB}$, for both HD and IM/DD techniques. We can see that $P_{\text {out }}$ is lower at $\mathrm{P}_{3}\left(\omega_{p}=0.24\right)$, while $\mathrm{P}_{2}$ and $\mathrm{P}_{1}$ respectively exhibit higher probabilities of failure.

(ii) $-\left\{\mathrm{P}_{3}, \mathrm{P}_{4}, \mathrm{P}_{5}, \mathrm{P}_{6}, \mathrm{P}_{7}\right\}$. As we move the RIS module from $\mathrm{P}_{3}$ to $\mathrm{P}_{7}$, we can observe from Figs. 9 and 10 that $P_{\text {out }}$ increases and the worse case corresponds to $\mathrm{P}_{6}\left(\omega_{p}=1.38\right)$. Note that this $P_{\text {out }}$ variation is not monotonic as from $\mathrm{P}_{6}, P_{\text {out }}$ starts decreasing again and the system exhibits a better $P_{\text {out }}$ at $\mathrm{P}_{7}$ when compared to $\mathrm{P}_{6}$. However, the $P_{\text {out }}$ result at $\mathrm{P}_{7}$ remains higher compared to that obtained at $\mathrm{P}_{3}$.

Figure 9 depicts $P_{\text {out }}$ versus $\bar{\gamma}_{\mathrm{eq}} / \gamma_{t h}$ for several PD's positions, $\varphi_{g, p}=\left\{0^{\circ}, 15^{\circ}, 30^{\circ}, 45^{\circ}, 60^{\circ}, 75^{\circ}\right\}$, considering two positions of the RIS, $\mathrm{P}_{3}\left(\varphi_{h, p}=0^{\circ}-\omega_{p}=0.24\right)$ and $\mathrm{P}_{6}$ $\left(\varphi_{h, p}=69.25^{\circ}-\omega_{p}=1.38\right)$. This is performed assuming that the receiver's field-of-view can accommodate these different positions. It can be observed, as predicted, that $P_{\text {out }}$ is lower for $\varphi_{g, p}=0^{\circ}$, and corresponds to the position where the incoming light ray forms a $90^{\circ}$ with the PD's surface. These results also show that a pattern is preserved between $\mathrm{HD}$ and IM/DD techniques.

(iii)- $\left\{\mathrm{P}_{7}, \mathrm{P}_{8}, \mathrm{P}_{9}\right\}$. We observe in Figs. 9 and 10 that from $\mathrm{P}_{7}$ $\left(\omega_{p}=2.61\right), P_{\text {out }}$ decreases and exhibits the lowest value at $\mathrm{P}_{9}$ for the proposed model.

\section{- Channel Capacity}

(i) $\left\{\mathrm{P}_{1}, \mathrm{P}_{2}, \mathrm{P}_{3}\right\}$. As in the case of $P_{\text {out }}$, Fig. 12 shows that as one moves from $\mathrm{P}_{3}$ to $\mathrm{P}_{1}$, the system's average channel capacity decreases for both HD and IM/DD techniques. This result is confirmed by Fig. 13 , where $\bar{C}$ is given versus $\omega_{p}$ for $\bar{\gamma}_{\text {eq }}=30 \mathrm{~dB}$ and $40 \mathrm{~dB}$, for both HD and IM/DD techniques. The system shows a higher channel capacity at $\mathrm{P}_{3}\left(\omega_{p}=0.24\right)$, while $\mathrm{P}_{2}$ and $\mathrm{P}_{1}$ respectively exhibit lower values.

(ii)- $\left\{\mathrm{P}_{3}, \mathrm{P}_{4}, \mathrm{P}_{5}, \mathrm{P}_{6}, \mathrm{P}_{7}\right\}$. When the RIS module is located between $P_{3}$ and $P_{7}$, we can see from Figs. 12 and 13 that 


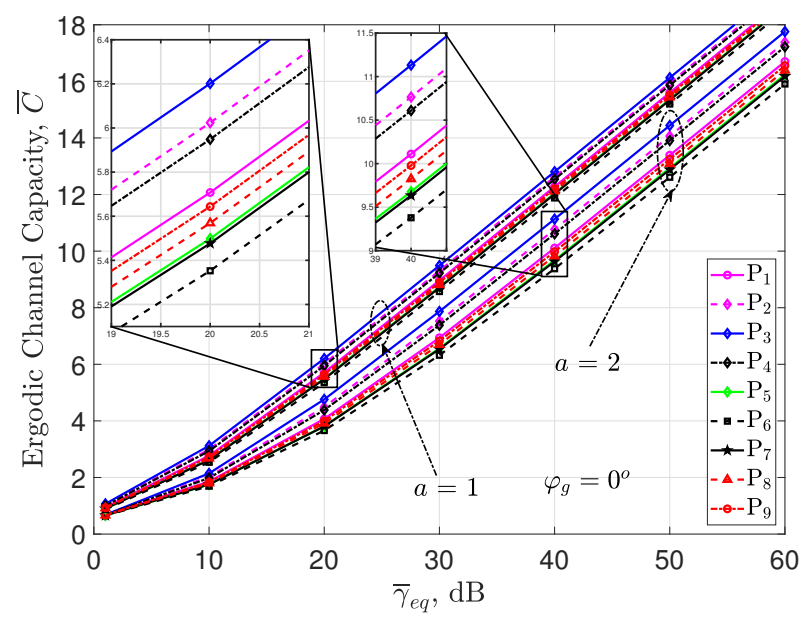

Fig. 12: Ergodic channel capacity, $\bar{C}$, versus $\bar{\gamma}_{\text {eq }}$ for several positions of the RIS module, when $\varphi_{g}=10^{\circ}, a=\{1,2\}$, and $\xi_{h}=1.1$.

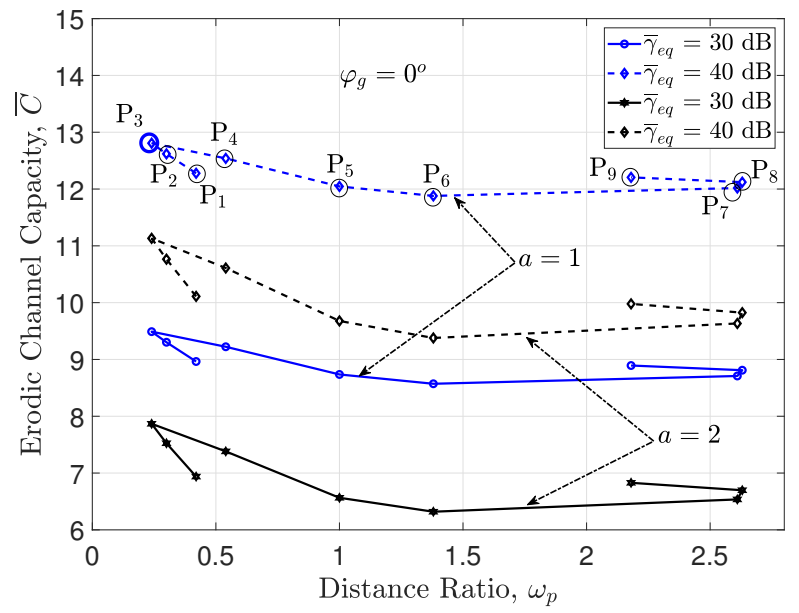

Fig. 13: Ergodic channel capacity, $\bar{C}$, versus $\omega_{p}$ for $\varphi_{g}=0^{\circ}$, $\bar{\gamma}_{\mathrm{eq}}=30 \mathrm{~dB}, 40 \mathrm{~dB}, a=\{1,2\}$, and $\xi_{h}=1.1$.

the average channel capacity decreases and the lowest value is obtained at $\mathrm{P}_{6}\left(\omega_{p}=1.38\right)$. Also as the in case of the OP, this variation of $\bar{C}$ is not monotonic as it tends to increase again from $P_{6}$, reaching a higher value at $P_{7}$. Nevertheless, its value at $P_{7}$ remains lower compared with the value at $P_{3}$.

Figure 14 gives the channel capacity versus $\bar{\gamma}_{\text {eq }}$ for several PD's positions, $\varphi_{g, p}=\left\{0^{\circ}, 15^{\circ}, 30^{\circ}, 45^{\circ}, 60^{\circ}, 75^{\circ}\right\}$, considering two positions of the RIS, $\mathrm{P}_{3}\left(\varphi_{h, p}=0^{\circ}-\omega_{p}=0.24\right)$ and $\mathrm{P}_{6}\left(\varphi_{h, p}=69.25^{\circ}-\omega_{p}=1.38\right)$. It can be observed that $\bar{C}$ is higher for $\varphi_{g, p}=0^{o}$, and it can also be seen that a pattern is preserved between HD and IM/DD techniques.

(iii) $-\left\{\mathrm{P}_{7}, \mathrm{P}_{8}, \mathrm{P}_{9}\right\}$. We can see in Figs. 12 and 13 that from $\mathrm{P}_{7}$ $\left(\omega_{p}=2.61\right), \bar{C}$ increases and exhibits the highest value at $\mathrm{P}_{9}$.

The top and bottom parts of Fig. 15 respectively depict analytical and simulation results of the OP, $P_{\text {out }}$, versus $\bar{\gamma}_{\text {eq }} / \gamma_{t h}$ and the ergodic channel capacity, $\bar{C}$, versus $\bar{\gamma}_{\text {eq }}$, at $\mathrm{P}_{3}$, $a=\{1,2\}$, and $\xi_{h, p}=1.1$. These results validate our derivation

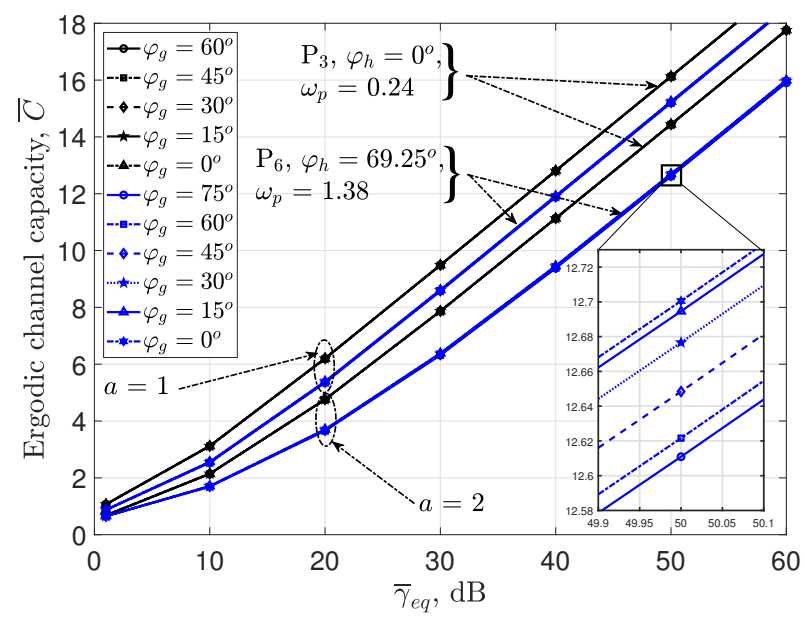

Fig. 14: Ergodic channel capacity, $\bar{C}$, versus $\bar{\gamma}_{\text {eq }}$ for $\varphi_{g}=\left\{0^{\circ}\right.$, $\left.15^{\circ}, 30^{\circ}, 45^{\circ}, 60^{\circ}, 75^{\circ}\right\}, \mathrm{P}_{3}$ and $\mathrm{P}_{6}, a=\{1,2\}$, and $\xi_{h, p}=$ 1.1 .
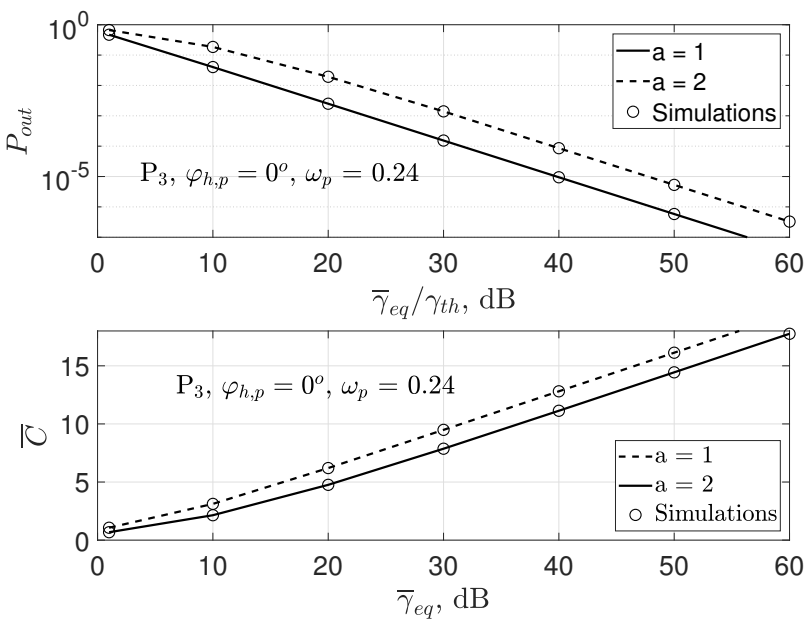

Fig. 15: Outage probability (analytical and simulation results), $P_{\text {out }}$, versus $\bar{\gamma}_{\mathrm{eq}} / \gamma_{t h}$ and Ergodic channel capacity (analytical and simulation results), $\bar{C}$, versus $\bar{\gamma}_{\text {eq }}$, at $\mathrm{P}_{3}, a=\{1,2\}$, and $\xi_{h, p}=1.1$.

of the expressions of OP and ergodic channel capacity.

\section{- Bit Error Rate (BER)}

(i) $-\left\{\mathrm{P}_{1}, \mathrm{P}_{2}, \mathrm{P}_{3}\right\}$. The patterns observed for the OP and average channel capacity are preserved for the BER. Figure 16 confirms that considering $\mathrm{P}_{1}, \mathrm{P}_{2}$, and $\mathrm{P}_{3}$, the system performs better at $\mathrm{P}_{3}$ for both $\mathrm{HD}$ and IM/DD techniques. This pattern is also depicted in Fig. 17 , where $\bar{P}_{b}$ is given in terms of $\omega_{p}$ for $\bar{\gamma}_{\text {eq }}=30 \mathrm{~dB}$ and $40 \mathrm{~dB}$, for both HD and IM/DD techniques. The system shows a lower probability of error at $\mathrm{P}_{3}\left(\omega_{p}=\right.$ 0.24), and the highest at $\mathrm{P}_{1}$.

(ii) $-\left\{\mathrm{P}_{3}, \mathrm{P}_{4}, \mathrm{P}_{5}, \mathrm{P}_{6}, \mathrm{P}_{7}\right\}$. Between $\mathrm{P}_{3}$ and $\mathrm{P}_{7}$, Figs. 16 and 17 show that the worse performance of the proposed system is obtained at $\mathrm{P}_{6}\left(\omega_{p}=1.38\right)$. Even though the system performance gets better as we move from $\mathrm{P}_{6}$ to $\mathrm{P}_{7}, \mathrm{P}_{3}$ still exhibits the best system performance. 


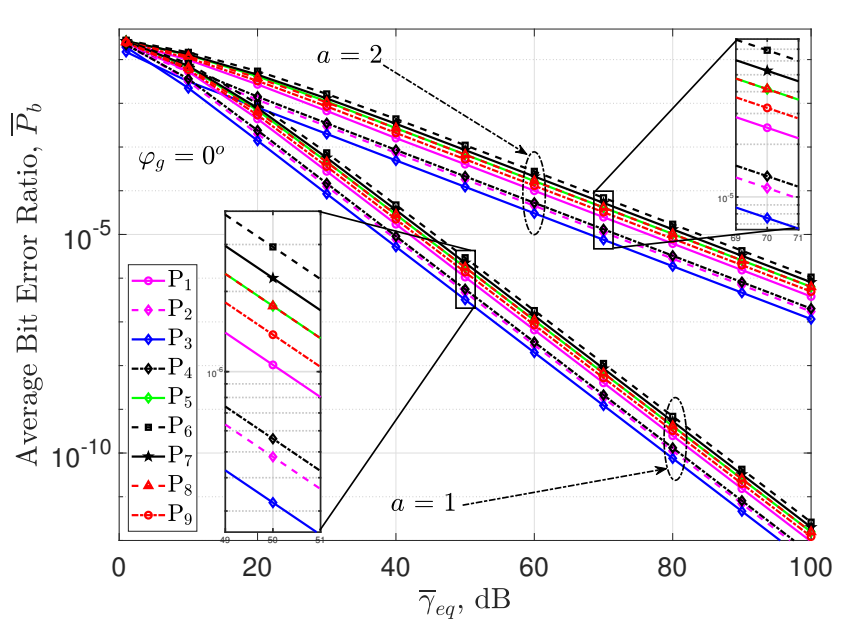

Fig. 16: BER, $\bar{P}$, versus $\bar{\gamma}_{\text {eq }}$ for several positions of the RIS module, when $\varphi_{g}=10^{\circ}, a=\{1,2\}$, and $\xi_{h}=1.1$.

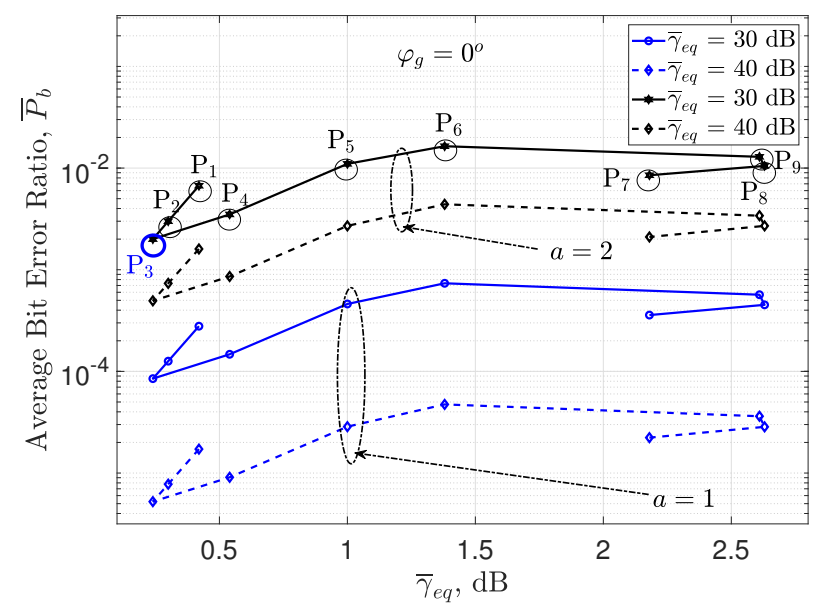

Fig. 17: BER, $\bar{P}$, versus $\omega_{p}$ for $\varphi_{g}=0^{o}, \bar{\gamma}_{\mathrm{eq}} / \gamma_{t h}=30 \mathrm{~dB}, 40$ $\mathrm{dB}, a=\{1,2\}$, and $\xi_{h}=1.1$.

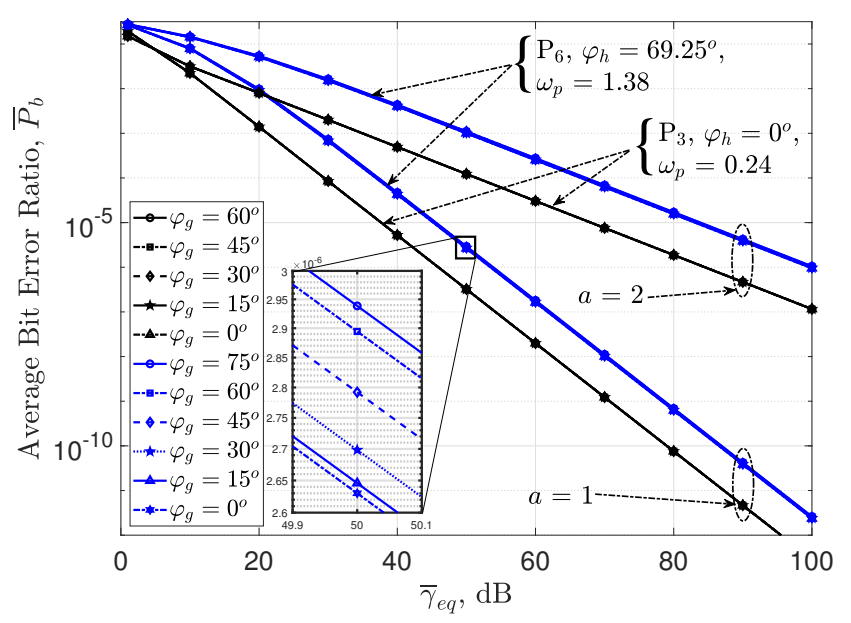

Fig. 18: BER, $\bar{P}$, versus $\bar{\gamma}_{\mathrm{eq}} / \gamma_{t h}$ for $\varphi_{g}=\left\{0^{\circ}, 15^{\circ}, 30^{\circ}, 45^{\circ}\right.$, $\left.60^{\circ}, 75^{\circ}\right\}, \mathrm{P}_{3}$ and $\mathrm{P}_{6}, a=\{1,2\}$, and $\xi_{h, p}=1.1$.

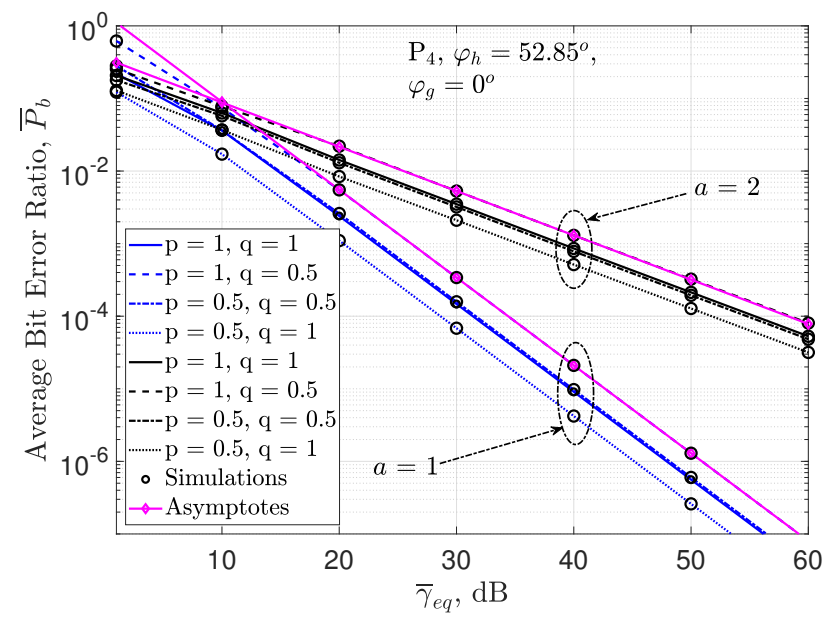

Fig. 19: Average BER of CBFSK, NBFSK, CBPSK, and DBPSK (analytical and simulation results) versus $\bar{\gamma}_{\mathrm{eq}}$ at $\mathrm{P}_{4}$ for $a=\{1,2\}$ and $\xi_{h, p}=1.1$.

In Fig. 18, we show $\bar{P}_{b}$ versus $\bar{\gamma}_{\text {eq }}$ for several PD's positions, $\varphi_{g, p}=\left\{0^{\circ}, 15^{\circ}, 30^{\circ}, 45^{\circ}, 60^{\circ}, 75^{\circ}\right\}$, considering two RIS positions, $\mathrm{P}_{3}\left(\varphi_{h, p}=0^{o}-\omega_{p}=0.24\right)$ and $\mathrm{P}_{6}\left(\varphi_{h, p}=\right.$ $\left.69.25^{\circ}-\omega_{p}=1.38\right)$. We observe that $\bar{P}_{b}$ is lower for $\varphi_{g, p}=$ $0^{\circ}$. It can also be seen that a pattern is preserved between HD and IM/DD techniques, considering $\mathrm{P}_{3}$ and $\mathrm{P}_{6}$.

(iii) $-\left\{\mathrm{P}_{7}, \mathrm{P}_{8}, \mathrm{P}_{9}\right\}$. Beyond $\mathrm{P}_{7}\left(\omega_{p}=2.61\right)$, we can see in Figs. 16 and 17 that $\bar{P}_{b}$ decreases and exhibits its lowest value at $\mathrm{P}_{9}$.

Finally, Fig. 19, obtained at $\mathrm{P}_{4}\left(\varphi_{h, p}=52.85^{\circ}-\omega_{p}=0.54\right)$, for $(p, q)=(1,1),(1,0.5),(0.5,0.5)$, and $(0.5,1)$, illustrates the asymptotic BER curves for selected schemes. The diversity gains are illustrated by their negative slopes. It can also be seen that simulation results match the analytical ones, confirming the accuracy of our derivation.

\section{- Summary}

The metrics used to evaluate the proposed system, which are the OP, the channel capacity, and the BER, reveal that the system performs better when the RIS module is closer to S; this corresponds to the lowest value of $\omega_{p}, 0.24$ in our case. These three metrics also show that beyond $\mathrm{P}_{1}$, the system performance worsens. On the contrary, beyond $\mathrm{P}_{9}$, they show that the system is likely to offer a good performance. However, this is not attractive for the following reasons: (i) Increasing $L_{h, p}$ also increases $L_{g, p}$, leading to a higher transmit power to overcome channel attenuation and losses for the same performance as that obtained at $\mathrm{P}_{3}$; (ii) Knowing that $\mathrm{S}$ and $\mathrm{D}$ are fixed points, considering the type and application of this system, which are respectively point-to-point and access network, it is not meaningful to go beyond points $\mathrm{P}_{1}$ and $\mathrm{P}_{9}$; and (iii) at large values of $L_{h, p}$ and $L_{g, p}$, we obtain $\omega_{p} \approx 1$ leading to a performance close to that of $\mathrm{P}_{5}$.

\section{CONCLUSION}

This paper has presented unified and Meijer-G functionbased closed-form expressions for the PDF, CDF, and MGF 
of a single-element RIS-based T-FSO link operating over G-G turbulence and pointing errors, considering several positions of the RIS module and the distance ratio, $\omega_{p}$. The beam waist and pointing displacement at the RIS and D are evaluated and used to investigate the system performance through the $\mathrm{OP}$, ergodic channel capacity, and average BER in terms of the distance ratio, $\omega_{p}$. The unified diversity order and coding gain for the proposed RIS-based T-FSO system have also been derived. It has been shown, through numerical results validated by simulation, that RIS-assisted T-FSO systems perform better when the RIS is closer to the transmitter.

\section{REFERENCES}

[1] I. S. Ansari, F. Yilmaz, and M. Alouini, "Performance analysis of freespace optical links over Málaga $(\mathcal{M})$ turbulence channels with pointing errors," IEEE Trans. Wireless Commun., vol. 15, no. 1, pp. 91-102, Jan. 2016.

[2] M. Zeng, X. Li, G. Li, W. Hao, and O. A. Dobre, "Sum rate maximization for IRS-assisted uplink NOMA," IEEE Commun. Lett., vol. 25, no. 1, pp. 234-238, Jan. 2021.

[3] M. A. ElMossallamy, H. Zhang, L. Song, K. G. Seddik, Z. Han, and G. Y. Li, "Reconfigurable intelligent surfaces for wireless communications: Principles, challenges, and opportunities," IEEE Trans. Cogn. Commun. Netw., vol. 6, no. 3, pp. 990-1002, Sep. 2020.

[4] S. Atapattu, R. Fan, P. Dharmawansa, G. Wang, J. Evans, and T. A. Tsiftsis, "Reconfigurable intelligent surface assisted two-way communications: Performance analysis and optimization," IEEE Trans. Commun., vol. 68 , no. 10 , pp. $6552-6567$, Oct. 2020.

[5] E. Basar, "Reconfigurable intelligent surface-based index modulation: A new beyond MIMO paradigm for 6G," IEEE Trans. Commun., vol. 68, no. 5, pp. 3187-3196, May 2020.

[6] J. Ye, S. Guo, and M. S. Alouini, "Joint reflecting and precoding designs for SER minimization in reconfigurable intelligent surfaces assisted MIMO systems," IEEE Trans. Wireless Commun., vol. 19, no. 8, pp. 5561-5574, Aug. 2020.

[7] A. R. Ndjiongue, T. M. N. Ngatched, O. A. Dobre, and H. Haas, "Towards the use of re-configurable intelligent surfaces in VLC systems: Beam steering," IEEE Wireless Commun. Mag., Apr. 2021, Accepeted.

[8] A. R. Ndjiongue, T. Ngatched, O. Dobre, and H. Haas, "Re-configurable intelligent surface-based VLC receivers using tunable liquid-crystals: The concept," IEEE/OSA J. Lightw. Technol., vol. 39, no. 10, pp. $3193-$ 3200, May 2021.

[9] P. P. Játiva, F. Seguel, and P. Adasme, "Evaluation of intelligent reflecting surfaces for diffuse visible light communications link," in IEEE Latin-American Conf. Commun. (LATINCOM), Santo Domingo, Dominican, 18-20 Nov. 2020, pp. 1-5.

[10] A. M. Abdelhady, A. K. S. Salem, O. Amin, B. Shihada, and M. S Alouini, "Visible light communications via intelligent reflecting surfaces: Metasurfaces vs mirror arrays," IEEE Open J. Commun. Soc., vol. 2, pp. 1-20, Dec. 2021.

[11] M. Najafi and R. Schober, "Intelligent reflecting surfaces for free space optical communications," in Proc. IEEE GLOBECOM Conf., Waikoloa, HI, USA, 9-13 Dec. 2019, pp. 1-7.

[12] M. Najafi, B. Schmauss, and R. Schober, "Intelligent reconfigurable reflecting surfaces for free space optical communications," IEEE Trans. Commun., pp. 1-1, May 2021, Early Access.

[13] H. Jia, J. Zhong, M. N. Janardhanan, and G. Chen, "Ergodic capacity analysis for FSO communications with UAV-equipped IRS in the presence of pointing error," in Proc. 20th IEEE Int. Conf. Commun. Technol. (ICCT), Nanning, China, 28-31 Oct. 2020, pp. 949-954.

[14] H. Ajam, M. Najafi, V. Jamali, and R. Schober, "Channel modeling for IRS-assisted FSO systems," ArXiv, Oct. 2020.

[15] M. Morales-Céspedes, M. C. Paredes-Paredes, A. García Armada, and L. Vandendorpe, "Aligning the light without channel state information for visible light communications," IEEE J. Sel. Areas Commun., vol. 36, no. 1, pp. 91-105, Jan. 2018.

[16] L. Yang, W. Guo, and I. S. Ansari, "Mixed dual-hop FSO-RF communication systems through reconfigurable intelligent surface," IEEE Commun Lett., vol. 24, no. 7, pp. 1558-1562, Jul. 2020.

[17] A. Sikri, A. Mathur, P. Saxena, M. R. Bhatnagar, and G. Kaddoum, "Reconfigurable intelligent surface for mixed FSO-RF systems with cochannel interference," IEEE Commun. Lett., pp. 1-1, Early Access, Feb. 2021.
[18] A. R. Ndjiongue, T. M. N. Ngatched, O. A. Dobre, and H. Haas, "Design of a power amplifying-RIS for free-space optical communication systems," arXiv, Apr. 2021.

[19] H. Wang, Z. Zhang, B. Zhu, J. Dang, L. Wu, L. Wang, K. Zhang, and Y. Zhang, "Performance analysis of multi-branch reconfigurable intelligent surfaces-assisted opticalwWireless communication system in environment with obstacles," IEEE Trans. Veh. Technol., Jun. 2021, Early Access.

[20] L. Yang, W. Guo, D. B. da Costa, and M.-S. Alouini, "Free-space optical communication with reconfigurable intelligent surfaces," ArXiv, Nov. 2020.

[21] R. Boluda-Ruiz, A. García-Zambrana, C. Castillo-Vázquez, B. CastilloVázquez, and S. Hranilovic, "Outage performance of exponentiated Weibull FSO links under generalized pointing errors," IEEE/OSA $J$. Lightw. Technol., vol. 35, no. 9, pp. 1605-1613, May 2017.

[22] A. Upadhya, V. K. Dwivedi, and G. K. Karagiannidis, "On the effect of interference and misalignment error in mixed RF/FSO systems over generalized fading channels," IEEE Trans. Commun., vol. 68, no. 6, pp. 3681-3695, Jun. 2020.

[23] W. Gappmair and H. E. Nistazakis, "Subcarrier PSK performance in terrestrial FSO links impaired by Gamma-Gamma fading, pointing errors, and phase noise," IEEE/OSA J. Lightw. Technol., vol. 35, no. 9 , pp. 1624-1632, May 2017.

[24] E. Zedini, H. Soury, and M. Alouini, "Dual-hop FSO transmission systems over Gamma-Gamma turbulence with pointing errors," IEEE Trans. Wireless Commun., vol. 16, no. 2, pp. 784-796, Feb. 2017.

[25] F. Benkhelifa, Z. Rezki, and M. Alouini, "Low SNR capacity of FSO links over Gamma-Gamma atmospheric turbulence channels," IEEE Commun. Lett., vol. 17, no. 6, pp. 1264-1267, Jun. 2013.

[26] Z. Yigit, E. Basar, and I. Altunbas, "Low complexity adaptation for reconfigurable intelligent surface-based MIMO systems," IEEE Commun. Lett., vol. 24, no. 12, pp. 2946-2950, Dec. 2020.

[27] L. Yang, F. Meng, J. Zhang, M. O. Hasna, and M. D. Renzo, "On the performance of RIS-assisted dual-hop UAV communication systems," IEEE Trans. Veh. Technol., vol. 69, Sep. 2020.

[28] D. Karp and E. Prilepkina, "Hypergeometric differential equation and new identities for the coefficients of Nørlund and Bühring," Symmetry, Integrability and Geometry: Methods and Appl., vol. 12, no. 52, pp. 1-23, May 2016. [Online]. Available: http://dx.doi.org/10.3842/ SIGMA.2016.052

[29] Wolfram Research, Inc., "Mathematica Edition-MeijerG, Version 8," Wolfram Research 2010, champaign, IL, USA.

[30] I. M. Ryzhik and I. S. Gradshtem, Table of Integrals, Series, and Products, 8th Edition. NY, USA: Academic Press, Sep. 2014.

[31] M. I. Petkovic, A. M. Cvetkovic, G. T. Djordjevic, and G. K. Karagiannidis, "Partial relay selection with outdated channel state estimation in mixed RF/FSO systems," IEEE/OSA J. Lightw. Technol., vol. 33, no. 13, pp. 2860-2867, Jul. 2015.

[32] L. Yang, F. Meng, Q. Wu, D. B. da Costa, and M. S. Alouini, "Accurate closed-form approximations to channel distributions of RISaided wireless systems," IEEE Wireless Commun. Lett., vol. 9, no. 11, pp. 1985-1989, Nov. 2020.

[33] M. Di Renzo, M. Iezzi, and F. Graziosi, "On diversity order and coding gain of multisource multirelay cooperative wireless networks with binary network coding," IEEE Trans. Veh. Technol., vol. 62, no. 3, pp. 11381157, Mar. 2013.

[34] M. D. Renzo, A. Guidotti, and G. E. Corazza, "Average rate of downlink heterogeneous cellular networks over generalized fading channels: A stochastic geometry approach," IEEE Trans. Commun., vol. 61, no. 7, pp. 3050-3071, Jul. 2013.

[35] N. A. M. Nor, E. Fabiyi, M. M. Abadi, X. Tang, Z. Ghassemlooy, and A. Burton, "Investigation of moderate-to-strong turbulence effects on free space optics - A laboratory demonstration," in 13th Int. Conf. Telecommun. (ConTEL), Graz, Austria, 13-15 Jul. 2015, pp. 1-5.

Alain R. Ndjiongue (S'14, M'18, SM'20) received the M.Eng. and D. Eng degrees in electrical and electronic engineering from the University of Johannesburg, South Africa, in 2013 and 2017, respectively. He served as a PDRF and senior lecturer at the same university from 2017 to 2020 and is currently a senior researcher at Memorial University of Newfoundland, Canada. His research interests are in digital communications, including power line and optical communications. He is an active reviewer for several prestigious IEEE ComSoc journals and conferences. He was a 2017 and 
2018 exemplary reviewer for IEEE Communication Letters, Optical Society of America, and a 2019 top 1\% peer reviewer in the Essential Science Indicators Research.

Telex. M. N. Ngatched (M'05, SM'17) received the B.Sc. degree and the M.Sc. degree in electronics from the University of Yaoundé, Cameroon, in 1992 and 1993, respectively, the MscEng (Cum Laude) in electronic engineering from the University of Natal, Durban, South Africa, in 2002, and the Ph.D. in electronic engineering from the University of KwaZuluNatal, Durban, South Africa, in 2006. From July 2006 to December 2007, he was with the University of KwaZulu-Natal as Postdoctoral Fellow, and from 2008 to 2012 with the Department of Electrical and Computer Engineering, University of Manitoba, Canada, as a Research Associate. He joined Memorial University in August 2012, where he is currently an Associate Professor and Coordinator of the Engineering One program at the Grenfell Campus.

His research interests include $5 \mathrm{G}$ and $6 \mathrm{G}$ enabling technologies, optical wireless communications, hybrid optical wireless and radio frequency communications, artificial intelligence and machine learning for communications, and underwater communications. He has authored over 100 refereed journal and conference papers in these areas.

Dr. Ngatched serves as an Associate Editor of the IEEE Open Journal of the Communications Society, an Editor of the IEEE Communications Society On-Line Content, and the Managing Editor of the IEEE Communications Magazine. He was the publication chair of the IEEE CWIT 2015, the Managing Editor of the IEEE Communications Letters from September 2018 to March 2020, an Associate Editor with the IEEE Communications Letters from 2015 to 2019, the co-chair of the Spectrum Management, Radio Access technology, Services and Security track of VTC2021-Spring and VTC2022Spring, and Technical Program Committee (TPC) member and session chair for many prominent IEEE conferences including GLOBECOM, ICC, WCNC, VTC, and PIMRC. He was a recipient of the Best Paper Award at the IEEE Wireless Communications and Networking Conference (WCNC) in 2019. He is a Professional Engineer (P. Eng.) registered with the Professional Engineers and Geoscientists of Newfoundland and Labrador, St. John's, NL, Canada.

Octavia A. Dobre (M'05, SM'07, F'20) received the Dipl. Ing. and Ph.D degrees from the Polytechnic Institute of Bucharest, Romania, in 1991 and 2000, respectively. Between 2002 and 2005, she was with New Jersey Institute of Technology, USA. In 2005, she joined Memorial University, Canada where she is currently a Professor and Research Chair. She was a Visiting Professor with Massachusetts Institute of Technology, USA and Université de Bretagne Occidentale, France. Her research interests encompass wireless, optical, and underwater communication technologies. She has (co-)authored over 350 refereed papers in these areas.

Dr. Dobre serves as the Editor-in-Chief (EiC) of the IEEE Open Journal of the Communications Society. She was the EiC of the IEEE Communications Letters, Senior Editor, Editor, and Guest Editor for various prestigious journals and magazines. She also served as General Chair, Technical Program CoChair, Tutorial Co-Chair, and Technical Co-Chair of symposia at numerous conferences.

Dr. Dobre was a Fulbright Scholar, Royal Society Scholar, and Distinguished Lecturer of the IEEE Communications Society. She obtained Best Paper Awards at various conferences, including IEEE ICC, IEEE Globecom, IEEE WCNC, and IEEE PIMRC. Dr. Dobre is a Fellow of the Engineering Institute of Canada and a Fellow of the Canadian Academy of Engineering. Ana Garcia Armada (S'96-A'98-M'00-SM'08) received the Ph.D. degree in electrical engineering from the Polytechnical University of Madrid in February 1998. She is currently a Professor at University Carlos III of Madrid, Spain, where she has occupied a variety of management positions (Head of Signal Theory and Communications Department, Vice-dean of Electrical Engineering, Deputy Vice-Chancellor of International Relations, among others). She is leading the Communications Research Group at this university. She has been visiting scholar at Stanford University, Bell Labs and University of Southampton. She has participated (and coordinated most of them) in more than 30 national and 10 international research projects as well as 20 contracts with the industry, all of them related to wireless communications. She is the co-author of eight book chapters on wireless communications and signal processing. She has published more than 150 papers in international journals and conference proceedings, and she holds four patents.
She has contributed to international standards organizations, such as ITU and ETSI, is member of the expert group of the European 5G PPP and member of the advisory committee 5JAC of the ESA as expert appointed by Spain on 5G. She has served on the editorial boards of Physical Communication (20082017), IET Communications (2014-2017), and IEEE Communications Letters (Exemplary Editor Award 2017 and 2018). She serves on the editorial board of and IEEE Transactions on Communications and IEEE Open Journal of the Communications Society.

She has served on the TPC of more than 40 conferences, and she has been/is part of the organizing committee of IEEE Globecom (2019, 2021 General Chair), IEEE Vehicular Technology Conference (VTC) Fall 2018, Spring 2018 and 2019 and IEEE 5G Summit 2017, among others. She was the Newsletter Editor of the IEEE ComSoc Signal Processing and Consumer Electronics Committee (2017-2018) and is now the Secretary of this committee (since 2019). She has been the Secretary of the IEEE ComSoc Women in Communications Engineering Standing Committee (2016-2017) and the Chair of this committee (2018-2019). She has received the Young Researchers Excellence Award, from University Carlos III of Madrid. She was awarded the third place Bell Labs Prize 2014 for shaping the future of information and communications technology. She received the Outstanding service award from the IEEE ComSoc Signal Processing and Communications Electronics (SPCE) technical committee in 2019 and from IEEE ComSoc Women in Communications Engineering Standing Committee in 2020. Her main interests are multi-carrier and multi-antenna techniques and signal processing applied to wireless communications.

Harald Haas (Fellow, IEEE) is the Director of the LiFi Research and Development Centre at the University of Strathclyde. He is also the Initiator, co-founder, and Chief Scientific Officer of pureLiFi Ltd. He received the $\mathrm{Ph} . \mathrm{D}$. degree from The University of Edinburgh in 2001. He has authored 550 conference and journal papers, including papers in Science and Nature Communications. Haas' main research interests are in optical wireless communications, hybrid optical wireless and RF communications, spatial modulation, and interference coordination in wireless networks. His team invented spatial modulation. He introduced LiFi to the public at an invited TED Global talk in 2011. This talk on Wireless Data from Every Light Bulb has been watched online over 2.72 million times. LiFi was listed among the 50 best inventions in TIME Magazine in 2011. He gave a second TED Global lecture in 2015 on the use of solar cells as LiFi data detectors and energy harvesters. This has been viewed online over 2.75 million times. In 2016, he received the Outstanding Achievement Award from the International Solid State Lighting Alliance. In 2019 he was recipient of IEEE Vehicular Society James Evans Avant Garde Award. Haas was elected a Fellow of the Royal Society of Edinburgh (RSE) in 2017. In the same year he received a Royal Society Wolfson Research Merit Award and was elevated to IEEE Fellow. In 2018 he received a threeyear EPSRC Established Career Fellowship extension and was elected Fellow of the IET. Haas was elected Fellow of the Royal Academy of Engineering (FREng) in 2019. 